my journal manuscript No.

(will be inserted by the editor)

\title{
Relativistic magnetic reconnection in pair plasmas and its astrophysical applications
}

\author{
D. Kagan · L. Sironi · B. Cerutti · \\ D. Giannios
}

Received: date / Accepted: date

\begin{abstract}
This review discusses the physics of magnetic reconnection - a process in which the magnetic field topology changes and magnetic energy is converted to kinetic energy - in pair plasmas in the relativistic regime. We focus on recent progress in the field driven by theory advances and the maturity of particle-in-cell codes. This work shows that fragmentation instabilities at the current sheet can play a critical role in setting the reconnection speed and affect the resulting particle acceleration, anisotropy, bulk flows, and radiation. Then, we discuss how this novel understanding of relativistic reconnection can be applied to high-energy astrophysical phenomena, with an emphasis on pulsars, pulsar wind nebulae, and active galactic nucleus jets.
\end{abstract}

Keywords acceleration of particles $\cdot$ galaxies: active $\cdot$ instabilities $\cdot$ magnetic reconnection · pulsars: general · radiation mechanisms: non-thermal · relativistic processes

All authors contributed equally to this review.

D. Kagan

Racah Institute of Physics, The Hebrew University of Jerusalem, Jerusalem 91904, Israel

Raymond and Beverly Sackler School of Physics and Astronomy, Tel Aviv University, Tel Aviv 69978, Israel

E-mail: daniel.kagan@astro.huji.ac.il

L. Sironi

Harvard-Smithsonian Center for Astrophysics, Cambridge, MA, 02138, USA

E-mail: lsironi@cfa.harvard.edu

B. Cerutti

Department of Astrophysical Sciences, Princeton University, Princeton, NJ 08544, USA

E-mail: bcerutti@astro.princeton.edu

D. Giannios

Department of Physics and Astronomy, Purdue University, 525 Northwestern Avenue, West Lafayette, IN, 47907, USA

E-mail: dgiannio@purdue.edu 


\section{Introduction}

Magnetic reconnection is a common phenomenon in which the topology of magnetic field lines is changed and magnetic energy is converted to kinetic energy. Interpretations of space plasma measurements (e.g., Chen et al. 2008, Øieroset et al. 2011) and astronomical observations suggest that reconnection occurs in many places in the Universe. Because the length scale of magnetic fields in astrophysical plasmas is extremely large, of order the size of astrophysical sources, while low plasma resistivity means that the characteristic scale of dissipation is very small, magnetic field lines are typically "frozen" into the astrophysical plasma, inhibiting dissipation. The topological change in the field lines produced by reconnection can break flux freezing and facilitate dissipative energy conversion.

In this review, we focus on reconnection in pair plasmas in the relativistic regime, in which the magnetic energy before the fields reconnect is significantly greater than the total enthalpy of the particles, so that the particles become relativistic when they enter the reconnection region. This condition is precisely stated as

$$
\sigma \equiv \frac{B^{2}}{4 \pi m n c^{2} w_{n}}>1
$$

where $B$ is the magnetic field, $n$ is the total particle number density including all species, and $w_{n}$ is the enthalpy per particle (assumed to be the same for both species), given by $w_{n}=\gamma+P /\left(m n c^{2}\right)$, where $\gamma$ is the mean particle Lorentz factor and $P$ is the particle pressure. 1

Relativistic reconnection may be of importance in astrophysical magnetically dominated systems such as Pulsar Wind Nebulae (PWN), as well as relativistic jets in Active Galactic Nuclei (AGN) or Gamma Ray Bursts (GRB) which may be magnetically dominated. The observed radiation from such systems is typically highly energetic and nonthermal. Because shock acceleration of particles through the Fermi process is likely to be inefficient in magnetically dominated flows (Sironi and Spitkovsky 2011b, Sironi et al. 2013), it is expected that reconnection is responsible for the acceleration of high energy particles and the production of radiation in these magnetically dominated systems. The role of relativistic reconnection in particle acceleration and radiation is a primary subject of this review.

This paper is organised as follows. In the remainder of Section 1, we review simple models of relativistic reconnection (Section 1.1) and discuss the physics of particle acceleration in relativistic reconnection (Section 1.2) In Section 2, we discuss simulations of relativistic reconnection and the resulting particle acceleration, anisotropies, and bulk flows. In Section 3 , we explore the application of relativistic reconnection in astrophysical systems; this section includes predictions of the radiation spectrum resulting from reconnection in those systems. Finally, in Section 4 we present our conclusions.

1 If $w_{n} \gg 1$ but $\sigma<1$, the plasma is initially relativistic but reconnection is typically weak, so the relativistic reconnection discussed in this review typically fulfils condition (1). 


\subsection{Models of Reconnection}

We now discuss models of reconnection in detail. Whenever regions of opposite magnetic polarity are present, Maxwell's equations imply that there will be a current sheet in between. In this current layer, magnetic field lines can diffuse across the plasma to reconnect at one or more X-lines. During reconnection, magnetized plasma approaches the central plane of the current layer with an asymptotic inflow velocity $v_{\text {in }}$, which is also known as the reconnection velocity. After passing the X-line, plasma is expelled from the vicinity of the X-line to either side at the outflow velocity $v_{\text {out }}$, which is typically assumed to equal the characteristic speed of magnetic disturbances in plasma, the Alfvén velocity $v_{\mathrm{A}}$. In the relativistic regime, $v_{\mathrm{A}}=c \sqrt{\sigma /(1+\sigma)} \sim c$. The dimensionless reconnection rate is usually defined as $r_{\text {rec }} \equiv v_{\text {in }} / v_{\text {out }}$.

Outside of the current sheet, non-ideal effects are negligible and the magnetohydrodynamic (MHD) condition

$$
\mathbf{E}+\frac{1}{c}\langle\mathbf{v}\rangle \times \mathbf{B}=0
$$

holds, where $\mathbf{E}$ is the electric field, $\mathbf{B}$ is the magnetic field, and $\langle\mathbf{v}\rangle$ is the mean particle velocity.

In a steady-state configuration which is quasi-two dimensional and does not vary strongly perpendicular to the plane of reconnection, the electric field throughout the reconnection region $\mathbf{E}_{\mathrm{rec}}$ is uniform and can be found by applying the condition (2) outside the current sheet, giving

$$
\mathbf{E}_{\mathrm{rec}}=-\frac{1}{c}\left(\mathbf{v}_{\mathrm{in}} \times \mathbf{B}_{0}\right),
$$

where $\mathbf{B}_{0}$ is the reversing magnetic field outside the current sheet. Because there is no velocity flow inside the current sheet, the electric field there is sustained by some non-ideal effect which is responsible for dissipation. The reconnection rate $r_{\text {rec }}$ may be related to the electric field by the equation

$$
r_{\text {rec }} \equiv \frac{v_{\text {in }}}{v_{\text {out }}}=\frac{E_{\text {rec }}}{\left(v_{\mathrm{A}} / c\right) B_{0}} .
$$

\subsubsection{Sweet-Parker resistive and kinetic relativistic reconnection}

Defining $\delta$ and $L$ to be the thickness and length of the current sheet, the conservation of mass from the reconnection inflow to the outflow in an incompressible plasma requires

$$
\frac{\delta}{L}=r_{\text {rec }}=\frac{v_{\text {in }}}{v_{\text {out }}} \sim \frac{v_{\text {in }}}{v_{\mathrm{A}}} .
$$

This equation is not always applicable to relativistic reconnection due to the possible presence of relativistic bulk flows which violate the incompressibility assumption, but it does apply in the simple steady-state models we discuss in this section. 
In the Sweet-Parker resistive model of reconnection, $L$ is taken to be the macroscopic length scale of the magnetic field, while the thickness $\delta$ is determined by the dissipation rate that can be sustained by resistivity. The dimensionless parameter that determines the importance of collisional resistivity is the Lundquist number $S \equiv v_{\mathrm{A}} L / \eta$, where $\eta$ is the magnetic diffusivity produced by resistivity. Lyubarsky (2005) has shown that the reconnection rate for relativistic Sweet-Parker resistive reconnection is

$$
r_{\text {rec }}=\frac{\delta}{L} \sim \frac{1}{\sqrt{S}},
$$

which is identical to the result for non-relativistic Sweet-Parker resistive reconnection. Since the Lundquist number $S$ is very large in astrophysical plasmas (depending on the application, $S \sim 10^{20}$ may be a typical value), Sweet-Parker reconnection is extremely slow. On the other hand, solar flares are believed to be powered by magnetic reconnection requiring that $v_{\text {in }} / v_{\mathrm{A}} \sim$ 0.1 !

Since the collisional resistivity is often extremely small in magnetically dominated astrophysical plasmas, kinetic effects resulting from individual particle motions are likely to be more important than resistivity in many systems.

The characteristic frequency of kinetic effects is the plasma oscillation frequency $\omega_{\mathrm{p}}$, given by

$$
\omega_{\mathrm{p}}=\sqrt{\frac{4 \pi n q^{2}}{w_{n} m}},
$$

where $q$ is the charge of the particles. Kinetic effects become important on spatial scales smaller than the corresponding inertial length $c / \omega_{\mathrm{p}}$ (also known as "skin depth"). Comisso and Asenjo (2014) have shown that when kinetic effects are important, the reconnection rate in the relativistic case is given by

$$
r_{\mathrm{rec}}=\frac{c}{\omega_{\mathrm{p}} L}
$$

Because $c / \omega_{\mathrm{p}}$ is small compared to the macroscopic scale $L$ of the field lines, steady-state Sweet-Parker kinetic reconnection is still relatively slow.

\subsubsection{Fast reconnection and the tearing and plasmod instabilities}

There have been many attempts to identify effects that would result in current sheets with smaller aspect ratios $L / \delta$, to allow for faster reconnection. The most basic of these models is the Petschek mechanism (Petschek 1964), which assumes that oblique slow shocks are present around a central X-point, and they effectively limit the length of the reconnection region. Simulations in the non-relativistic regime have found that this configuration is unstable unless an anomalous localised resistivity is present in the center of the reconnection layer, i.e., at the X-line (Uzdensky and Kulsrud 2000). If the aspect ratio of the reconnection region is larger than $\sim 100$, oblique slow shocks can form at the end of the reconnection exhausts, (Liu et al. 2012; Higashimori and 
Hoshino 2012), but it is uncertain whether these shocks are analogous to those in the Petschek model. Despite the difficulty in confirming the viability of this mechanism, the name "Petschek reconnection" is often used to describe fast reconnection because kinetic effects can produce an effective anomalous resistivity. Below, we occasionally use the relativistic "Petschek" model derived by Lyubarsky (2005) to parameterise the properties of fast reconnection in the relativistic regime.

Most other models of fast reconnection focus on the effects of instabilities in the current layer. In any current sheet, the oppositely oriented fields constitute a source of free energy. An important instability that draws on this energy is the tearing instability, which at the same time mediates and is mediated by reconnection. The tearing instability produces an alternating series of narrow $\mathrm{X}$-lines where reconnection can occur, separated by large flux ropes. In turn, steady reconnection equilibria contain thin current sheets, which themselves can be unstable to the tearing instability. The nonideal effect that violates flux freezing to produce reconnection at these X-lines may be collisional resistivity, or it may arise from kinetic effects, so the tearing instability, like reconnection, can take both resistive and kinetic forms. The growth rate of the tearing instability depends strongly on the width of the current sheet. For fast growth, the sheet width must be comparable to those associated with resistive or kinetic reconnection (Biskamp 2000, Pétri and Kirk 2007).

A Sweet-Parker resistive current sheet is thin enough that a resistive instability of the Sweet-Parker current sheet, called the plasmodia instability, may break the sheet into X-lines and magnetic islands, thus lowering its aspect ratio $L / \delta$ and leading to relatively fast reconnection rates $r_{\text {rec }} \sim 0.01$ even at high Lundquist numbers, for which the unperturbed Sweet-Parker reconnection would be extremely slow (Loureiro et al. 2007: Samtaney et al. 2009: Huang and Bhattacharjee 2010). However, the corresponding reconnection rate in the relativistic case is significantly lower, $r_{\text {rec }} \sim 0.0001$ (Zanotti and Dumbser 2011). In a long kinetic current sheet whose width is comparable to the skin depth, the kinetic tearing instability can grow quickly and break up the current sheet into X-lines and flux ropes, which can result in fast reconnection at $r_{\text {rec }} \sim 0.1$ (e.g., Birn and Hesse 2001). A phase diagram of reconnection has been proposed uniting Sweet-Parker and plasmoid configurations for resistive and kinetic reconnection, with the transition from resistive to kinetic reconnection occurring when the Sweet-Parker sheet width approaches the skin depth, and the transition from Sweet-Parker to plasmoid configurations occurring as the aspect ratio of the reconnection region increases (Ji and Daughton 2011; Comisso and Asenjo 2014). The transition between resistive and kinetic regimes has been proposed as a possible explanation of observed variability in reconnection sites (Goodman and Uzdensky 2008) and the onset of fast reconnection far from the central engine in a Poynting flux model of GRBs (McKinney and Uzdensky 2012). In this review, we focus on the study of kinetic relativistic reconnection and the particle acceleration and radiation that can be produced by such reconnection, because kinetic effects will often 
dominate in relativistic magnetically dominated astrophysical plasmas, which are typically nearly collisionless.

\subsection{Particle acceleration and radiation in reconnection}

As discussed earlier, it is thought that magnetic reconnection is likely to be responsible for the acceleration of particles in systems that are magnetically dominated. As particles cross the current sheet at the X-line, they are forced to return into the current sheet by the reversing magnetic field, following Speiser orbits (Speiser 1965). Particles following such orbits can be accelerated in the direction perpendicular to the plane of reconnection (e.g. Zenitani and Hoshino 2001) by the reconnection electric field. Other acceleration mechanisms, in both X-lines and flux ropes, have been found in kinetic simulations for a review of these mechanisms see Oka et al. (2010), as well as the discussion in Section 2.3

The energy gain per unit time for a charged particle accelerated electromagnetically is given in general by

$$
\frac{d W}{d t}=q \mathbf{E} \cdot \mathbf{v} \sim q E c,
$$

Particles accelerated in relativistic magnetically dominated systems are typically thought to radiate via the synchrotron mechanism, which tends to place a fundamental constraint on the maximum energy of electromagnetically accelerated particles. The total synchrotron power emitted by a particle is given approximately by

$$
\frac{d W}{d t} \sim \frac{2 q^{4} B^{2} \gamma^{2}}{3 m^{2} c^{3}} .
$$

In regions where the MHD condition (2) holds, $E \leq B$ and setting $E=B$ allows the derivation of a maximum $\gamma$ for charged particles, which corresponds to a maximum radiation frequency referred to as the synchrotron burnoff limit. However, during reconnection particles experiencing extreme acceleration at the X-line can spend most of their time deep in the reconnection layer where $E>B$ (Uzdensky et al. 2011). Thus, they are able to evade this restriction and produce radiation beyond the burnoff limit, as we demonstrate in Section 3.1 ,

\section{Particle-in-cell simulations of reconnection}

\subsection{Numerical setup}

\subsubsection{Numerical techniques}

The most common method for simulating the kinetic dynamics of a reconnecting plasma involves the use of a particle-in-cell (PIC) code that evolves 
the discretized equations of electrodynamics - Maxwell's equations and the Lorentz force law. See Birdsall and Langdon (1991) for a detailed discussion of this method. PIC codes can model astrophysical plasmas from first principles, as a collection of charged macro-particles that are moved by integration of the Lorentz force. Each macroparticle represents many physical particles. Currents associated with the macro-particles are deposited on a grid on which Maxwell's equations is discretized. Electromagnetic fields are then advanced via Maxwell's equations, with particle currents as the source term. Finally, the updated fields are extrapolated to the particle locations and used for the computation of the Lorentz force, so the loop is closed self-consistently. So long as current deposition is the only effect of the macro-particles on the field quantities, charge conservation is ensured. This approach is capable of treating all effects present in collisionless plasmas, including particle acceleration to high energies. To ensure that kinetic effects are resolved in the simulation, it is necessary that the grid spacing be much smaller than the skin depth $c / \omega_{\mathrm{p}}$, and that the timestep be much smaller than the corresponding timescale $\omega_{\mathrm{p}}^{-1}$. To ensure that the momentum space distribution is adequately sampled, keep particle noise at a low level, and reduce the effects of unphysical collisions due to the relatively small number of particles in a Debye sphere, it is necessary that there be several particles per cell for each particle species.

\subsubsection{The Harris current sheet}

The starting equilibrium of most reconnection simulations is the Harris current sheet, which is an exact 1D equilibrium of plasma physics (Harris 1962). It is characterised by the field profile

$$
\mathbf{B}=B_{0} \tanh \frac{y}{\delta} \hat{\mathbf{x}}+\kappa B_{0} \hat{\mathbf{z}},
$$

where $\delta$ is the half-thickness of the current sheet, which must be of the same order as $c / \omega_{\mathrm{p}}$ for fast reconnection to occur. The quantity $\kappa$ sets the relative strength of a uniform "guide" field (orthogonal to the reconnection plane) which may be present in realistic reconnection configurations. For most of the discussion below, we will assume $\kappa=0$ for the sake of simplicity.

The particles within the current sheet in the Harris equilibrium are initialised in a drifting Maxwell-Juttner thermal distribution in which positively and negatively charged particles have equal and opposite bulk velocities $\boldsymbol{\beta}_{+}=$ $-\boldsymbol{\beta}_{-}=\boldsymbol{\beta}$ (in units of the speed of light) and drifting Lorentz factors of $\gamma_{d}=1 / \sqrt{1-\beta^{2}}$.

The density profile of the Harris current sheet including both electrons and positrons in the simulation frame is

$$
n=n_{0} \operatorname{sech}^{2} \frac{y}{\delta},
$$

Pressure equilibrium requires that $B_{0}^{2}=8 \pi n_{0} T_{0}$, where $T_{0}$ is the temperature of the particles (in units of $m c^{2}$ ) in the current sheet including the 


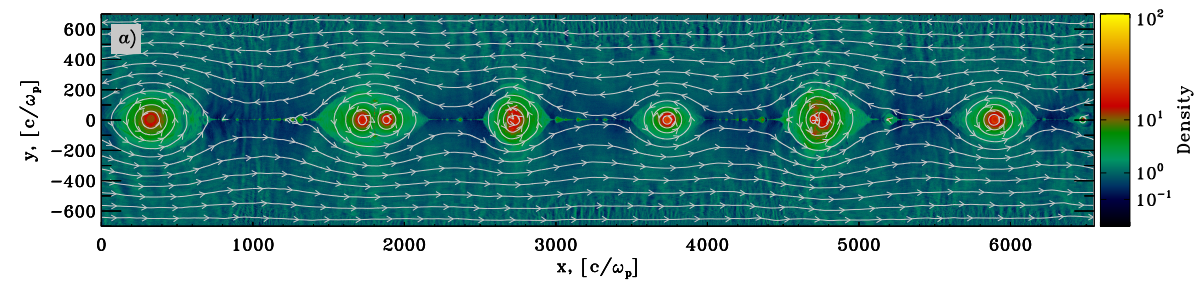

Fig. 1 Structure of the particle density in the reconnection layer at $\omega_{\mathrm{p}} t=3000$, from a $2 \mathrm{D}$ simulation of $\sigma=10$ reconnection presented in Sironi and Spitkovsky (2014).

Boltzmann constant in the simulation frame. Ampère's Law requires that

$$
\boldsymbol{\beta}_{+}=-\boldsymbol{\beta}_{-}=B_{0} /\left(4 \pi n_{0} q \delta\right)(-\hat{\mathbf{z}}) .
$$

This simple configuration is unstable to the tearing instability and is useful for studying reconnection. An additional uniform background population of particles with rest-frame density $n_{b}$ and no drift velocity is typically added to the current sheet population. Thus, the total density in the simulation frame of all particles in the middle of the current sheet is $n_{0}+n_{\mathrm{b}}$, whereas the total density in the background plasma away from the current sheet is $n_{\mathrm{b}}$. Using the expression for pressure equilibrium above allows us to express the value of $\sigma$ far from the current sheet as

$$
\sigma=\frac{2 n_{0} T_{0}}{n_{b} w_{n, \mathrm{~b}}} .
$$

where $w_{n, b}$ is the mean enthalpy of the particles in the background plasma. Note that the value of $n_{0} T_{0}$ is a Lorentz invariant. This equilibrium can be modified while retaining the same value of $\sigma_{b}$ by increasing the temperature $T_{0}$ and decreasing the value of $n_{0} / n_{b}$ to produce an equilibrium with less density contrast but a difference in temperature between the populations; for a detailed discussion, see Melzani et al. (2013). This modification is used in the simulations in this paper.

While the Harris sheet is the most common initial condition for studying reconnection, it should be mentioned that there are other possibilities. Reconnection can be initialised using a force-free current sheet (Guo et al. 2014), and dynamical scenarios such as X-point collapse (Graf von der Pahlen and Tsiklauri 2014). Finally, fully three dimensional configurations (Pontin 2011, and references therein) are likely to be the most realistic starting points for simulation, but only a few PIC simulations have used such configurations (Baumann and Nordlund 2012; Olshevsky et al. 2013).

\subsection{Structure of the reconnection layer}

We now present the structure and the dynamics of the reconnection layer, discussing the results of $2 \mathrm{D}$ and $3 \mathrm{D}$ PIC simulations. We concentrate on the 


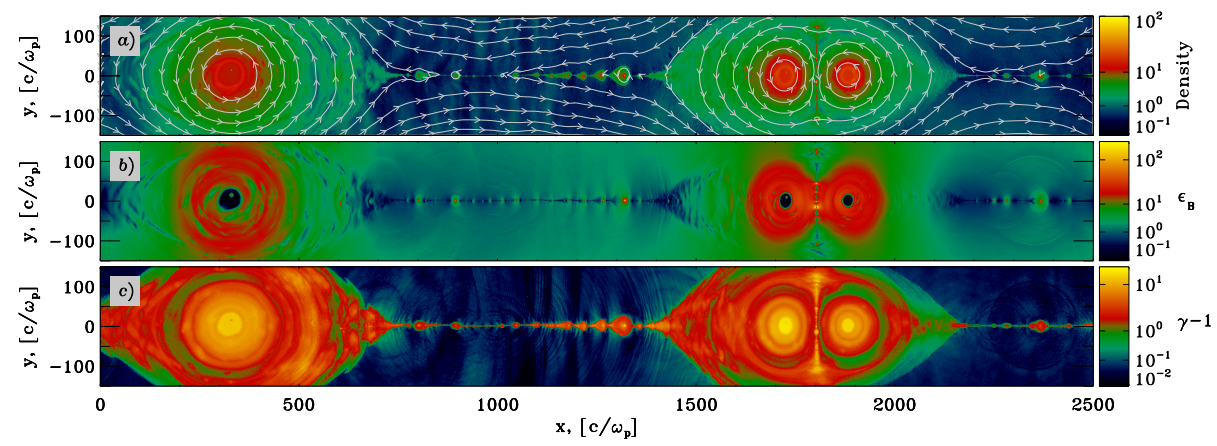

Fig. 2 Structure of the reconnection layer at $\omega_{\mathrm{p}} t=3000$, from a $2 \mathrm{D}$ simulation of $\sigma=10$ reconnection discussed in Sironi and Spitkovsky (2014). This figure is a zoom-in at $0 \leq$ $x \leq 2500 \mathrm{c} / \omega_{\mathrm{p}}$ of Fig. 1. We present (a) particle density, in units of the number density far from the current sheet (with overplotted magnetic field lines), (b) magnetic energy fraction $\epsilon_{B}=B^{2} / 8 \pi m n_{\mathrm{b}} c^{2}$ and (c) mean kinetic energy per particle.

case of an electron-positron plasma, which has been most widely explored in the literature, both in 2D (Zenitani and Hoshino 2001, 2005, 2007: Zenitani and Hesse 2008; Jaroschek et al. 2004, 2008, Bessho and Bhattacharjee 2005, 2007, 2012; Daughton and Karimabadi 2007, Lyubarsky and Liverts |2008; Cerutti et al. 2012b, 2013 Werner et al. 2014) and in 3D (Zenitani and Hoshino 2008; Yin et al. |2008; Liu et al. 2011; Sironi and Spitkovsky |2011a, 2012, Kagan et al. |2013, Cerutti et al. 2014b; Sironi and Spitkovsky 2014, Guo et al. 2014). The physics of relativistic electron-proton reconnection, yet still at an early stage of investigation, shows remarkable similarities with electronpositron reconnection (Melzani et al. 2014).

As described above, the reconnection layer is set up in Harris equilibrium, with the magnetic field reversing at $y=0$. For the sake of simplicity, we discuss here the case of anti-parallel fields, without a guide field component. The strength of the alternating fields is parameterized by the magnetization $\sigma$ defined in Eq. 1. Here, we assume that the background plasma far from the current sheet is cold, so $w_{n} \sim 1$ and $\sigma=B_{0}^{2} / 4 \pi m n_{\mathrm{b}} c^{2}$.

As a result of the tearing instability, the reconnection layer fragments into a series of magnetic islands (or flux tubes), separated by X-points. Over time, the islands coalesce and grow to larger scales (Daughton and Karimabadi 2007 have described a similar evolution in non-relativistic reconnection). The structure of the reconnection region at late times is presented in Fig. 1, from a largescale $2 \mathrm{D}$ simulation in a $\sigma=10$ pair plasma presented in Sironi and Spitkovsky (2014). By zooming into the region $0 \lesssim x \lesssim 2500 c / \omega_{\mathrm{p}}$ (here, the inertial length $c / \omega_{\mathrm{p}}$ is measured taking the density far from the current sheet), we see that each X-line is further fragmented into a number of smaller islands. This is a result of the secondary tearing mode (or "plasmoid instability") discussed by Uzdensky et al. (2010). The secondary islands lie at $700 c / \omega_{\mathrm{p}} \lesssim x \lesssim 1400 c / \omega_{\mathrm{p}}$ in Fig. 2, They are overdense (Fig. 2 a), filled with hot particles (Fig. 22) and 

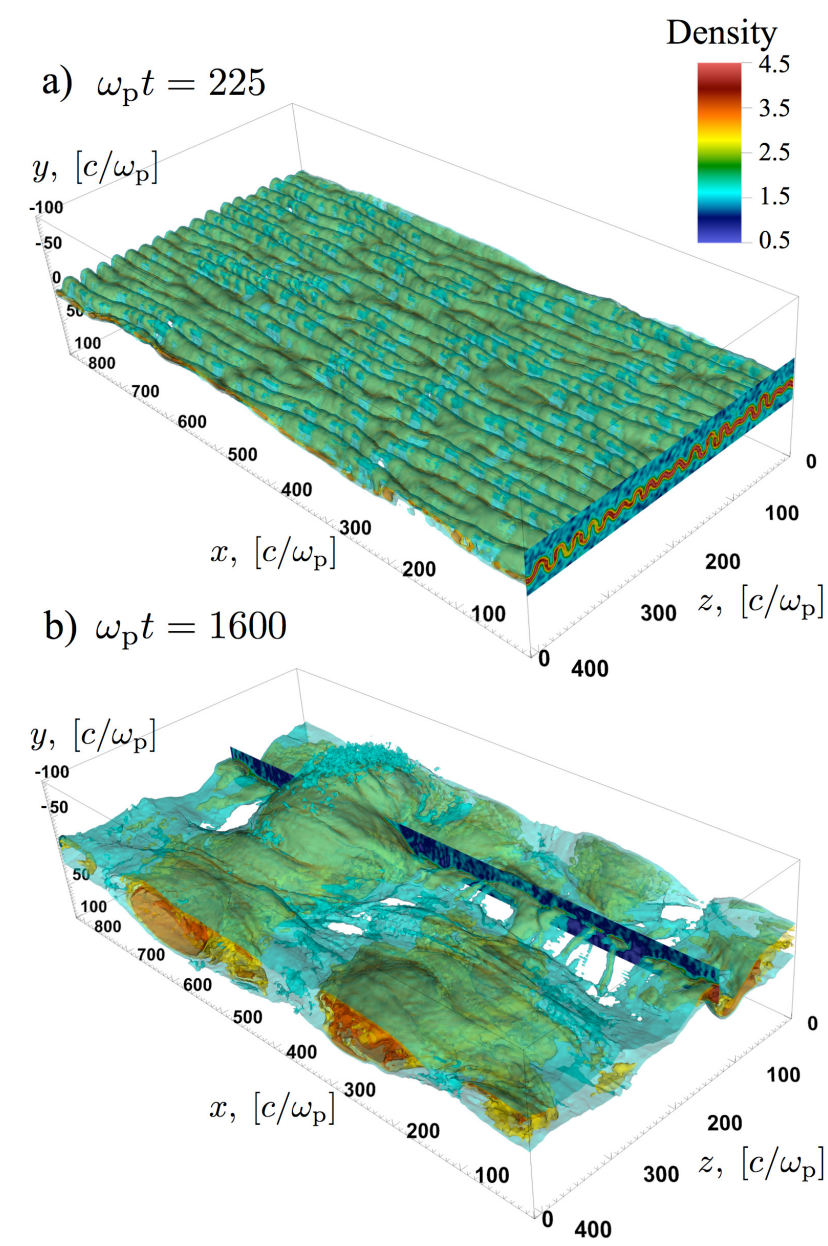

Fig. 3 Structure of the particle density at two different times: (a) $\omega_{\mathrm{p}} t=250$ and (b) $\omega_{\mathrm{p}} t=1600$. The plot refers to a 3D simulation of $\sigma=10$ reconnection without a guide field, presented in Sironi and Spitkovsky (2014). The 2D slices in the top and bottom panels (at $x=0$ and $z=130 \mathrm{c} / \omega_{\mathrm{p}}$, respectively) show the particle number density in that plane.

confined by strong fields (Fig. 2b). In between each pair of secondary islands, a secondary X-point mediates the transfer of energy from the fields to the particles. As shown in the next section, efficient particle acceleration occurs at the X-points.

The reconnection rate is $r_{\text {rec }} \equiv v_{\text {in }} / v_{\text {out }} \sim v_{\text {in }} / c \simeq 0.08$ for $\sigma=10$, nearly constant at late times. The reconnection rate depends on the plasma magnetization. In the case of vanishing guide field, Sironi and Spitkovsky (2014) quote that the reconnection rate in $2 \mathrm{D}$ increases from $r_{\mathrm{rec}} \simeq 0.03$ for $\sigma=1$ to $r_{\text {rec }} \simeq 0.12$ for $\sigma=30$, and it is nearly independent of $\sigma$ for larger magnetizations, in agreement with the analytical model by Lyubarsky (2005). 
After entering the current sheet, the flow is advected towards the large magnetic islands by the tension force of the reconnected magnetic field (in Fig. $2 \mathrm{p}$-c, the major islands are at $200 c / \omega_{\mathrm{p}} \lesssim x \lesssim 500 c / \omega_{\mathrm{p}}$ and $1600 c / \omega_{\mathrm{p}} \lesssim$ $\left.x \lesssim 1900 c / \omega_{\mathrm{p}}\right)$. Pushed by the ram pressure of the reconnection outflows, the major islands move along the layer, merging with neighboring islands. A merger event in indeed seen at $x \sim 1800 c / \omega_{\mathrm{p}}$ in Fig. 2. The current sheet formed between the two merging islands is unstable to the tearing mode, and it breaks into a series of secondary islands along the $y$ direction (orthogonal to the primary current sheet).

The evolution of $3 \mathrm{D}$ reconnection at late times parallels closely the $2 \mathrm{D}$ physics described above, even in the absence of a guide field 2 As shown in Fig. 3 a , the early phases of evolution are governed by the so-called driftkink (DK) mode (Zenitani and Hoshino 2008: Cerutti et al. 2014b; Sironi and Spitkovsky 2014). The DK instability corrugates the current sheet in the $z$ direction, broadening the layer and inhibiting the growth of the tearing mode at early times. However, at later times the evolution is controlled by the tearing instability (Sironi and Spitkovsky 2014), that produces in the $x y$ plane a series of magnetic islands (or rather, flux tubes), in analogy to the 2D physics. The reconnection layer at late times is organized into a few major islands (see the overdense plasmoids in Fig. $3 \mathrm{~b}$ ), separated by underdense regions (transparent in Fig. 3b) where field dissipation by reconnection is most efficient. In short, at late times the $3 \mathrm{D}$ physics parallels closely the $2 \mathrm{D}$ evolution presented above (yet, with a smaller reconnection rate, $r_{\text {rec }} \simeq 0.02$ in $3 \mathrm{D}$ versus $r_{\text {rec }} \simeq 0.08$ in 2D). As discussed in the next section, this has important implications for the acceleration performance of relativistic reconnection in 3D.

\subsection{Non-thermal particle acceleration}

Relativistic reconnection is an efficient source of non-thermal particles. In Fig. 4 we present the time evolution of the particle energy spectrum, from a 2D simulation of reconnection with $\sigma=10$ performed by Sironi and Spitkovsky (2014). A generic by-product of relativistic reconnection is the generation of a broad non-thermal spectrum extending to ultra-relativistic energies. For $\sigma=10$, the spectrum at $\gamma \gtrsim 1.5$ can be fitted with a power-law of slope $p \equiv-d \log N / d \log \gamma \sim 2$ (dotted red line). The spectrum clearly departs from a Maxwellian distribution with mean energy $(\sigma+1) m c^{2}$ (red dashed line, which assumes complete field dissipation). As shown in Fig. 6. the power-law slope depends on the flow magnetization, being harder for higher $\sigma(p \sim 1.5$ for $\sigma=50$, compare solid and dotted green lines). The slope is steeper for lower magnetizations ( $p \sim 4$ for $\sigma=1$, solid and dotted black lines), approaching the result of non-relativistic reconnection, yielding poor acceleration efficiencies (Drake et al. 2010). In the limit $\sigma \gg 1$, Guo et al. (2014) and Werner et al.

2 The presence of a strong guide field orthogonal to the reconnecting plane guarantees that the $3 \mathrm{D}$ physics will resemble the $2 \mathrm{D}$ results, see Guo et al. (2014). 


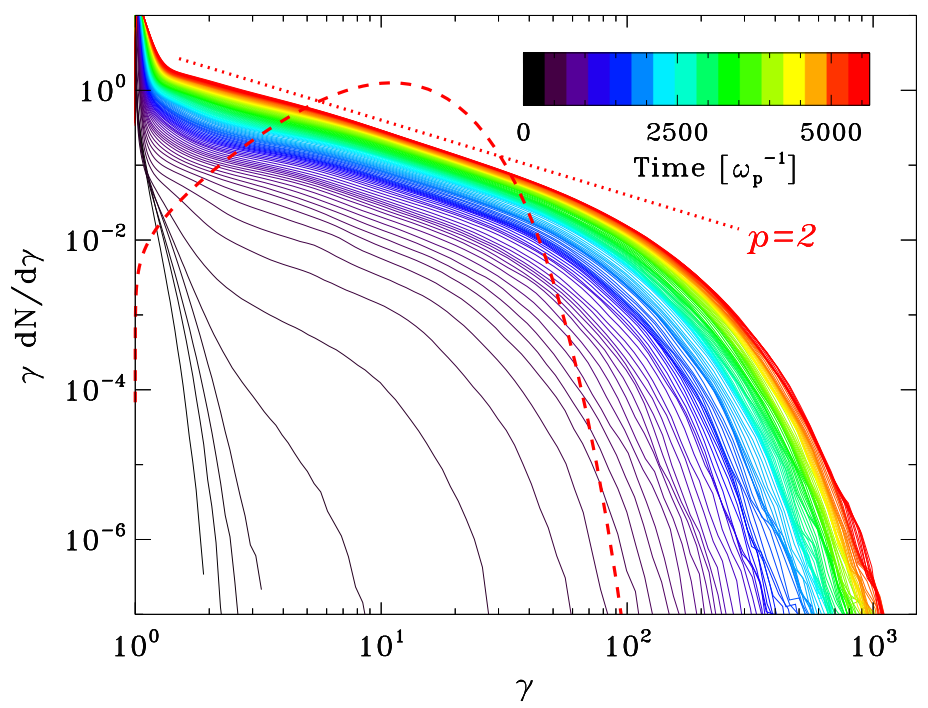

Fig. 4 Temporal evolution of the particle energy spectrum, from a 2D simulation of $\sigma=10$ reconnection by Sironi and Spitkovsky (2014). The spectrum at late times resembles a powerlaw with slope $p=2$ (dotted red line), and it clearly deviates from a Maxwellian with mean energy $(\sigma+1) m c^{2}$ (dashed red line, which assumes complete field dissipation).

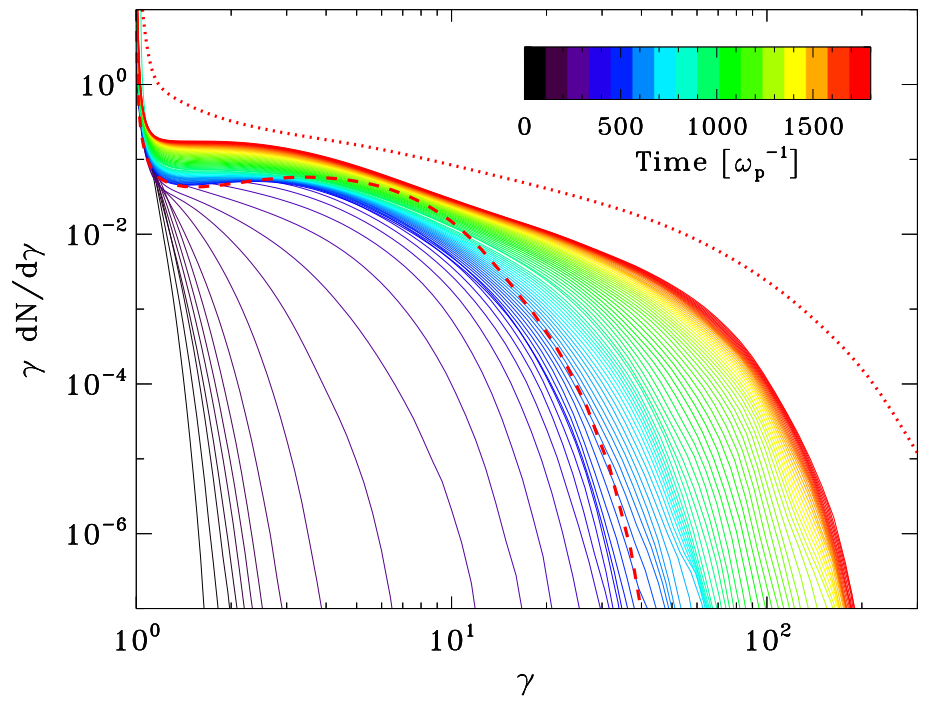

Fig. 5 Temporal evolution of the particle energy spectrum, from a 3D simulation of $\sigma=10$ reconnection by Sironi and Spitkovsky (2014). The spectra from two 2D simulations with in-plane (out-of-plane, respectively) anti-parallel fields are shown with red dotted (dashed, respectively) lines. 


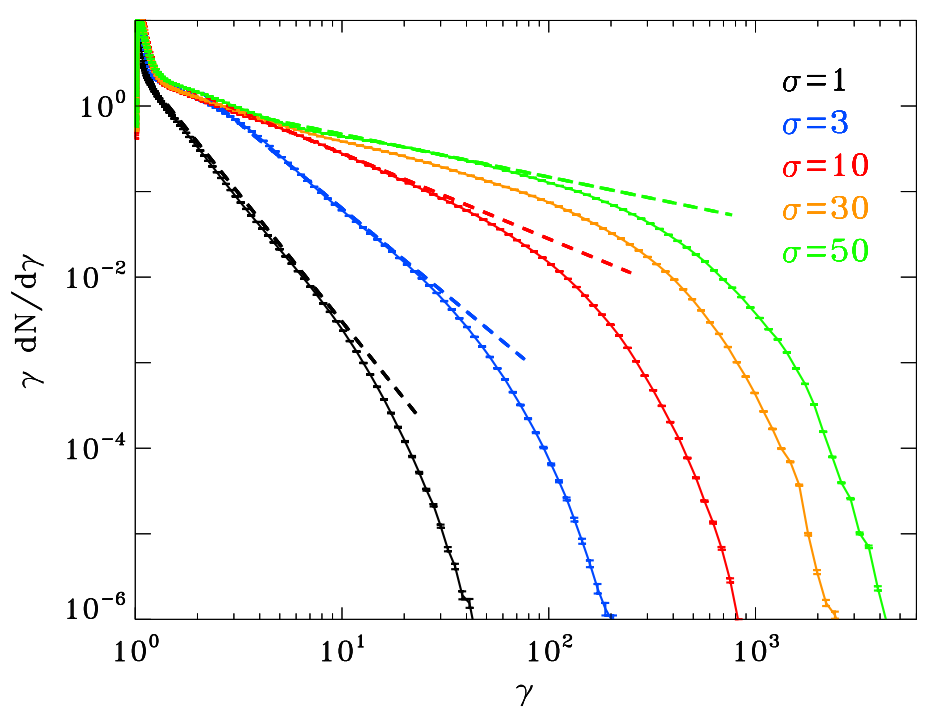

Fig. 6 Dependence of the spectrum on the magnetization, as indicated in the legend. The dotted lines refer to power-law slopes of $-4,-3,-2$ and -1.5 (from black to green).

(2014) have confirmed the trend described above, arguing that the non-thermal slope asymptotes to $p \simeq 1$ for highly magnetized flows.

For magnetizations $\sigma \gtrsim 10$ that yield $p \lesssim 2$, the increase in maximum energy over time is expected to terminate, since the mean energy per particle cannot exceed $(\sigma+1) m c^{2}$. For a power-law of index $1<p<2$ starting from $\gamma_{\min }=1$, the maximum Lorentz factor should saturate at $\gamma_{\max } \sim[(\sigma+1)(2-$ $p) /(p-1)]^{1 /(2-p)}$. For $\sigma \lesssim 10$ (where $p \gtrsim 2$ ), the increase in maximum energy does not stop, but it slows down at late times.

In short, 2D simulations of relativistic reconnection produce hard populations of non-thermal particles. However, the structure of $\mathrm{X}$-points in $3 \mathrm{D}$ is different from 2D, as emphasized in the previous section. In particular, the DK mode is expected to result in heating, not in particle acceleration (Zenitani and Hoshino 2007). Fig. 5 presents the temporal evolution of the particle spectrum in a 3D simulation with $\sigma=10$, by Sironi and Spitkovsky (2014). The spectrum at early times is quasi-thermal (black to blue lines in Fig. 5), and it resembles the distribution resulting from the DK mode (the red dashed line shows the spectrum from a 2D simulation with out-of-plane anti-parallel fields, to target the contribution of the DK mode). As discussed above, the DK mode is the fastest to grow, but the sheet evolution at late times is controlled by the tearing instability, in analogy to the $2 \mathrm{D}$ physics with in-plane fields. In fact, the spectrum at late times (cyan to red lines in Fig. 5 ) presents a pronounced high-energy power-law. The power-law slope is $p \sim 2.3$, close to the $p \sim 2$ index of $2 \mathrm{D}$ simulations with in-plane fields. With respect to the 
2D spectrum (dotted red line in Fig. 55, the normalization and the upper energy cutoff of the 3D spectrum are smaller, due to the lower reconnection rate $\left(r_{\text {rec }} \simeq 0.02\right.$ in $3 \mathrm{D}$ versus $r_{\text {rec }} \simeq 0.08$ in $\left.2 \mathrm{D}\right)$, so that fewer particles enter the current sheet per unit time, where they get accelerated by a weaker electric field $E_{\text {rec }} \sim r_{\text {rec }} B_{0}$.

The mechanism of particle acceleration at X-points has been the subject of various investigations, with analytical (Larrabee et al. 2003 Bessho and Bhattacharjee 2012) or numerical methods ${ }^{3}$ Using test particle simulations in prescribed electromagnetic fields, Nodes et al. (2003); Uzdensky et al. (2011); Cerutti et al. (2012a) found that reconnection naturally produces beams of high-energy particles aligned with the reconnection electric field present within the current layer. These particles follow relativistic Speiser orbits as they are moving back and forth across the reconnection layer. For a steady Sweet-Parker configuration, Uzdensky et al. (2011) showed that the meandering width of the Speiser orbit decreases as the energy of the particle increases, i.e., the most energetic particles, with larger Lorentz factor, are also the most focused along the electric field (see also Kirk 2004: Contopoulos 2007). The properties of these special orbits are also well captured by PIC simulations (Cerutti et al. 2012a, 2013). Fig. 7 shows the trajectory of a sample of 150 particles chosen randomly in a 2D PIC simulation with $\sigma=10$. The particle orbits are projected in the plane perpendicular to the reconnecting field, i.e., here in the $(y z)$-plane (reconnection happens in the $x y$-plane). Away from the two layers (located at $y / \rho_{\mathrm{c}} \sim 125$ and 375 , with $\left.\rho_{\mathrm{c}}=m c^{2} / e B_{0}\right)$, the particles are well magnetized: they gyrate along the field lines and remain at $z=0$. In contrast, the particles that enter the layer are efficiently boosted along the direction of the electric field (the $z$-axis) and follow relativistic Speiser orbits. The further the particle gets along the $z$-direction, the more energetic it will be.

The trajectories of a sample of particles extracted from a $2 \mathrm{D}$ simulation with $\sigma=10$ (in Fig. 8 from Sironi and Spitkovsky (2014)) also illustrate the mechanism for the formation of the power-law tail in the particle spectrum. At the X-point located at $x \sim 135 c / \omega_{\mathrm{p}}$ the magnetic energy is smaller than the electric energy (blue region in Fig. $8 \mathrm{p}$ ), so the particles become unmagnetized and they get accelerated along $z$ by the reconnection electric field. The final energy of the particles - the color in Fig. 8 a indicates the Lorentz factor measured at the outflow boundary of the X-line - directly correlates with the location at the moment of interaction with the current sheet (as argued in the

3 Particle acceleration in magnetic islands (as opposed to X-lines or X-points) is also widely discussed in the literature, both in non-relativistic reconnection (e.g., Drake et al. 2006 Oka et al. 2010 - where the particles are adiabatic, and they bounce several times between the two edges of an island - and relativistic reconnection (Liu et al. 2011, Guo et al. 2014), where the energy gain might come just from a single bounce. However, the inflowing particles interact at first with the X-points, where they get energy from the dissipating fields. It is this first acceleration episode (that we describe below) which will establish the spectral slope and strongly affect the future history of the inflowing particles. In fact, particles accelerated to high energies at the X-point are likely to experience further acceleration via reflection off of moving magnetic disturbances (e.g., in contracting islands or in between two merging islands), which might eventually dominate the overall energy gain. 


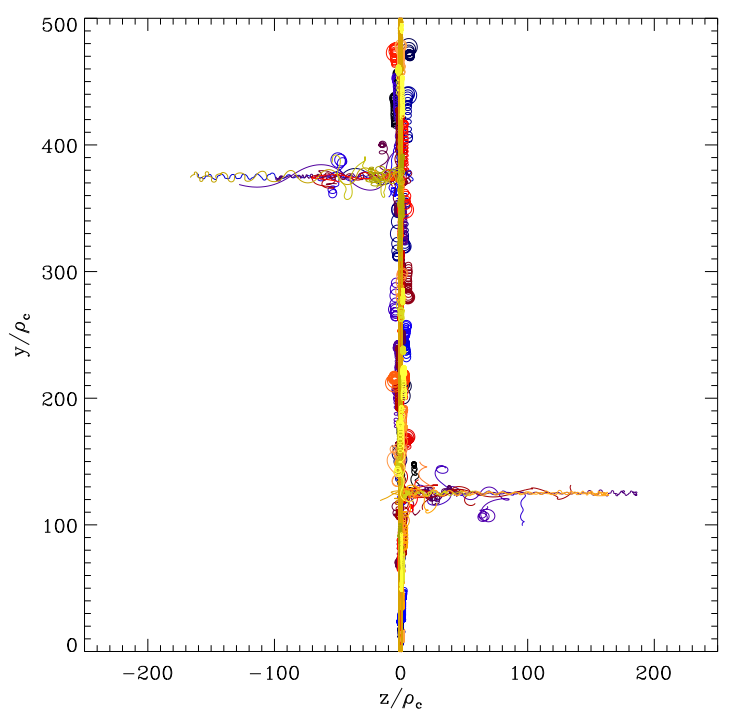

Fig. 7 Trajectories of a sample of 150 particles projected in the $(y z)$-plane from a 2D PIC simulation of relativistic reconnection with $\sigma=10$, and without guide field. Each orbit are drawn with a different color to increase the readability of this figure. The simulation starts with two anti-parallel Harris sheets of temperature $k T=m c^{2}$ located at $y / \rho_{\mathrm{c}} \sim 125$ and 375, where $\rho_{\mathrm{c}}=m c^{2} / e B_{0}$. Particles are accelerated along the $z$-axis within the current layers where the electric field is maximum, and they follow special orbits known as relativistic Speiser orbits. The further the particle gets along the $z$-axis, the more energetic the particle will become.

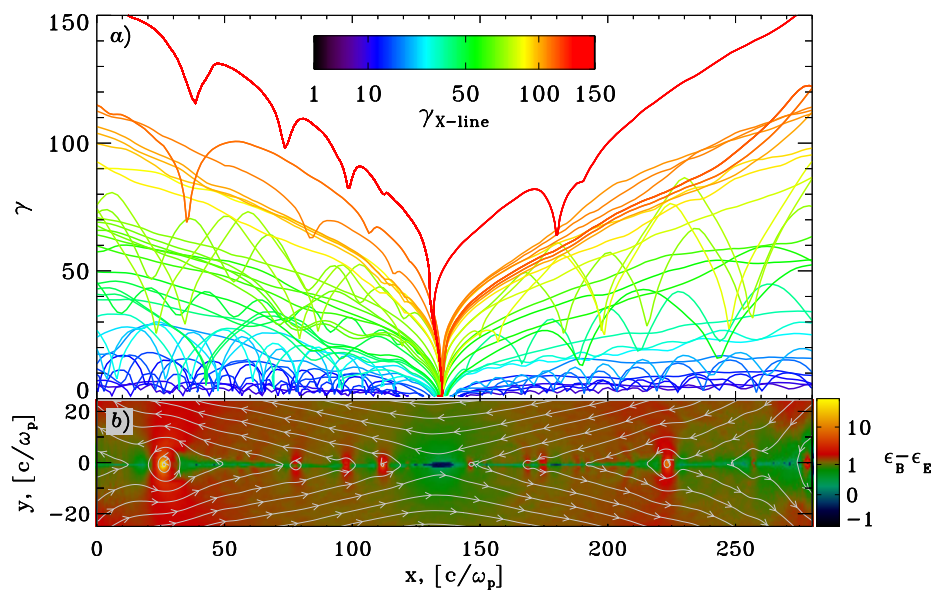

Fig. 8 (a) Energy evolution of a sample of selected particles interacting with a major Xpoint, as a function of the location $x$ along the current sheet. Colors are scaled with $\gamma_{\mathrm{X}-l i n e}$, the Lorentz factor attained at the outflow boundary of the X-line (at $x=0$ or $280 c / \omega_{\mathrm{p}}$ depending on the particle). (b) $\epsilon_{B}-\epsilon_{E}$ at the time when the particles interact with the $\mathrm{X}$-point (here, $\epsilon_{E}=E^{2} / 8 \pi m n_{\mathrm{b}} c^{2}$ is the electric energy fraction). 
analytical models by Larrabee et al. 2003; Bessho and Bhattacharjee 2012). Particles interacting closer to the center of the X-point (darkest blue in Fig. $8 \mathrm{~b}$ ) are less prone to be advected away along $x$ by the reconnected magnetic field, so they can stay longer in the acceleration region and reach higher Lorentz factors (orange and red lines in Fig. 8a). In other words, energetic particles turn slowly into the reconnected field ( $B_{y}$ in Fig. 8), because the Larmor radius is proportional to $\gamma$, so that they spend even more time at the $\mathrm{X}$-point than particles with lower energies. This is an argument originally proposed by Zenitani and Hoshino (2001), that may also explain the power-law nature of the spectrum (along with the impact parameter of the particles in the current sheet). Indeed, a broad power-law distribution is then established, as a result of the different energy histories of particles interacting at different distances from the X-point.

We point out that the most energetic particles (red and orange curves in Fig. 8) are slowly turning around the reconnected magnetic field $B_{y}$, and still have a positive $q \boldsymbol{E} \cdot \boldsymbol{v}$, so that they gain energy even outside of the blue region (where $|\boldsymbol{E}|>|\boldsymbol{B}|$ ). On the other hand, the green and blue particles experience also the electric fields surrounding the secondary islands, which explains the oscillations in their energy curves.

\subsection{Particle anisotropy and bulk motions}

It is now well established that relativistic reconnection is an efficient source of non-thermal particle acceleration (see previous section). In usual astrophysical environments, these energetic particles would emit non-thermal radiation via, e.g., synchrotron or inverse Compton scattering. Due to relativistic aberrations, the radiation emitted by highly relativistic particles (with $\gamma \gg 1$ ) is beamed within a cone of semi-aperture angle $\sim 1 / \gamma \ll 1$ along the direction of motion of the emitting particle. As a result, any anisotropy in the particle distribution results in an anisotropic distribution of radiation which is of critical importance in astronomy because the observer probes only one direction at a time. The overall energetic budget or even the shape of the particle spectrum inferred from observations could differ significantly from the isotropically averaged quantities.

Fig. 9 presents the angular distribution of the particle 4 -velocity vectors as a function of the particle energy, from a 2D PIC simulation with $\sigma \approx 10$ and with no guide field as first reported by Cerutti et al. (2012b). The low-energy particles $(\gamma \sim 1$, top panel) present little anisotropy because these particles have not been accelerated at X-points. At higher energies $(\gamma \gtrsim \sigma$, middle and bottom panel), the particles exhibit clear sign of anisotropy with two beams pointing roughly towards the $\pm x$-directions, i.e., along the reconnection exhausts. Hence, the beams are not necessarily pointing along the reconnection electric field because the tension of the reconnected field lines pushes the particles away from the X-points in the form of a reconnection outflow towards the magnetic islands (see Fig. 2a, and top panel in Fig. 11). Nonetheless, the 

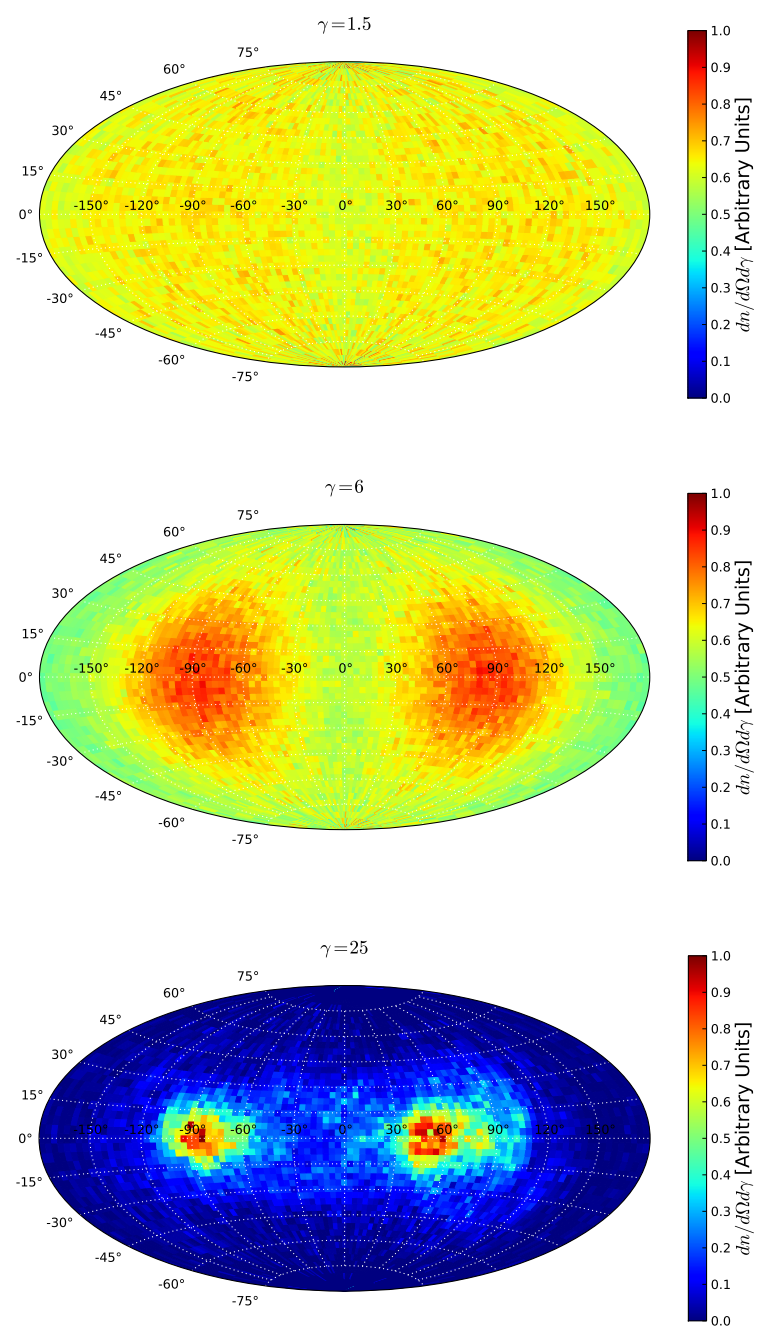

Fig. 9 Angular distribution of the particle 4 -velocity vectors $\mathbf{u}, d \mathrm{n} / d \Omega d \gamma$ (contour plot), in three energy bins: $\gamma=\mathbf{1 . 5} \pm \mathbf{0 . 1}$ (top), $\gamma=\mathbf{6} \pm \mathbf{0 . 3}$ (middle), and $\gamma=\mathbf{2 5} \pm \mathbf{1 . 2}$ (bottom). In this projection (Aitoff), each direction is given by the latitude angle $\left(\sin \phi=u_{\mathrm{y}} /|u|\right.$ with $-90^{\circ}<\phi<+90^{\circ}$, vertical axis) and the longitude angle $\left(\cos \lambda=u_{\mathrm{z}} / \sqrt{u_{\mathrm{x}}^{2}+u_{\mathrm{z}}^{2}}\right.$ with $-180^{\circ}<\lambda<+180^{\circ}$, horizontal axis). The precise geometry of the simulation is shown in Fig. 11 These results were obtained from a 2D PIC simulation with $\sigma=10$ with no guide field (see also Cerutti et al. 2012b, 2013 and Cerutti et al. 2014b in 3D). 


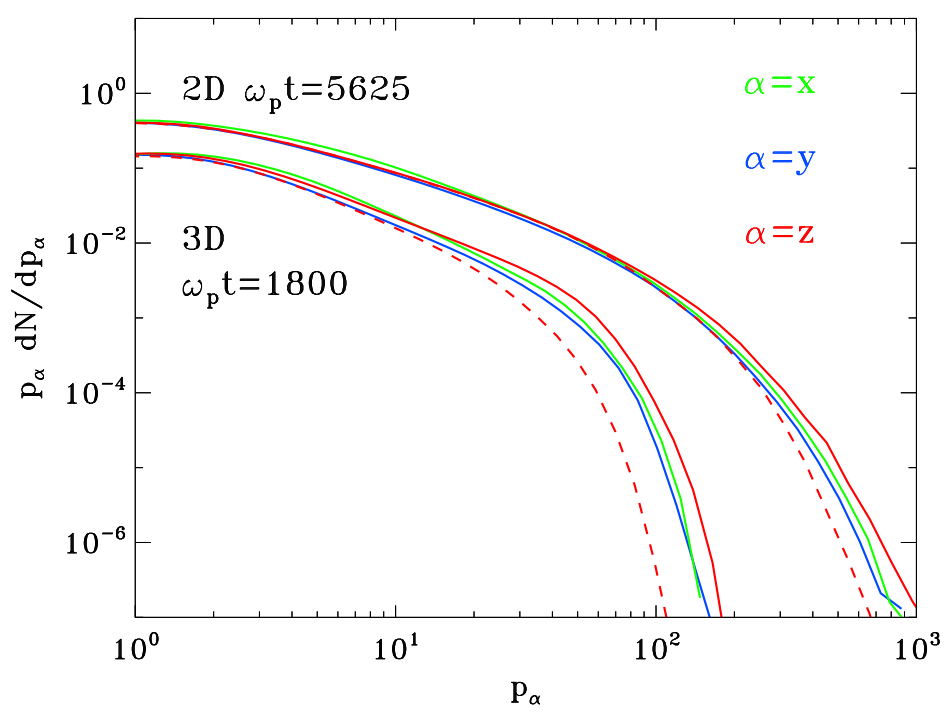

Fig. 10 Positron momentum spectrum along $x$ (green), $y$ (blue), $+z$ (red solid) and $-z$ (red dashed), for 2D and 3D, as indicated in the legend.

direction of the beam of energetic particles is not static: it wiggles rapidly within the $(x z)$-plane (along the horizontal axis in Fig. 9), which results in rapid flares of energetic radiation when the beam crosses the line of sight of a distant observer (Cerutti et al. 2012b). This result has interesting application to astrophysical flares, and in particular to the recently discovered $>100 \mathrm{MeV}$ gamma-ray flares discovered in the Crab Nebula (Cerutti et al. 2013, 2014b) (see Sect. 3.1). The Crab flare case is quite extreme in the sense that the particles emitting $>100 \mathrm{MeV}$ synchrotron radiation should be accelerated and radiating over a sub-Larmor timescale, so the highest energy radiation should keep the imprint of the particle anisotropy (regardless of the acceleration process), while the low-energy radiation should be more isotropic.

The pronounced anisotropy discussed above lasts for some limited amount of time. Indeed, when the high-energy particles reach the magnetic islands, they isotropize quickly in the strong fields shown in Fig. 2e and they do not contribute to the beamed emission. Since most of the particles at late times are contained in the major islands, it is not surprising that the long-term momentum spectra show little signs of anisotropy (see Fig. 10). Even the residual difference between the momentum spectra along $+z$ and $-z$ (red solid and dashed lines, respectively) diminishes at later times (the 2D momentum spectra at $\omega_{\mathrm{p}} t=1800$ were similar to the $3 \mathrm{D}$ results in Fig. 10, showing that the anisotropy decays over time).

It is important to stress that this beaming mechanism is strongly energydependent. It should be distinguished from the Doppler boosting due to a rel- 

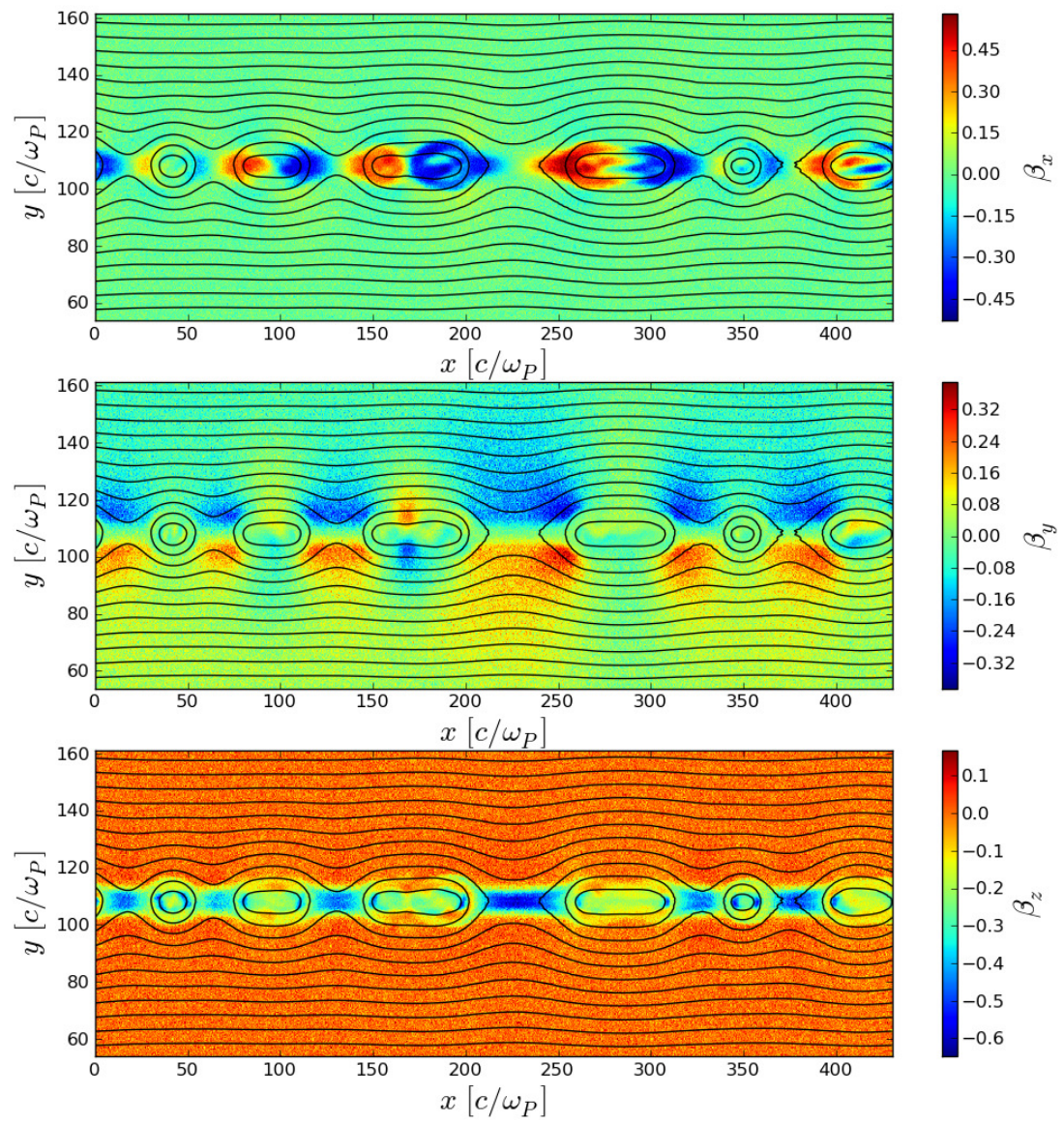

Fig. 11 Positron fluid velocity $\boldsymbol{\beta}=\mathbf{v} / c$ in the $x$-(top), $y$ - (middle), and $z$-directions (bottom), for the same simulation as in Fig. $9(\sigma=10$, no guide field). The black solid lines show the magnetic field lines. The electron fluid velocity maps are identical, except that $\beta_{\mathrm{z}, \text { electrons }}=-\beta_{\mathrm{z}, \text { positrons }}$.

ativistic bulk motion in the flow which beams all the particles and radiation by the same factor. In fact, relativistic reconnection produces also relativistic bulk flows as anticipated by Lyubarsky (2005), and constitutes the cornerstone of the fast-variability models for blazar jets by Giannios et al. (2009) (see Sect. 3.2. Fig. 11 shows the three components of the fluid velocity vector normalized by the speed of light, $\boldsymbol{\beta}=\mathbf{v} / c$, for the same simulation as in Fig. 9 (where $\sigma=10$ and with no guide field) and at the same stage. The $x$-component presents the characteristic signature of a dipolar relativistic flow 


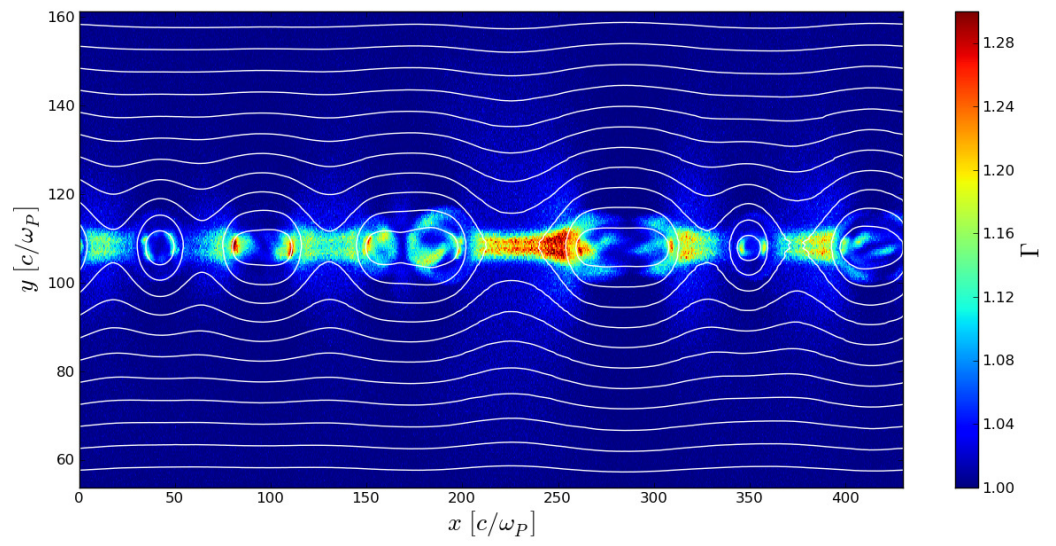

Fig. 12 Total Lorentz factor of the positron fluid, $\Gamma=1 / \sqrt{1-\beta^{2}}$, computed from Fig. 11 The white solid lines are magnetic field lines.

at every $\mathrm{X}$-point where $\beta_{\mathrm{x}} \approx \pm 0.5$, which corresponds to the reconnection outflow accelerated by the tension of the newly reconnected field lines (i.e., $v_{\text {out }} / c$ defined in Sect. 1.1). The $y$-component shows the inflow of particles from the upstream towards the X-point that feeds the reconnection process with fresh plasma (i.e., $v_{\text {in }} / c$ in Sect. 1.1). This motion is due to the $\mathbf{E}_{\mathrm{z}} \times \mathbf{B}_{\mathrm{x}}$ drift velocity, and is about $\beta_{\mathrm{y}} \approx \pm 0.3$ in this particular simulation. The $z$ component is related to the electric current carried by counter-streaming electrons and positrons around the $\mathrm{X}$-points. The corresponding fluid velocity is about $\beta_{\mathrm{z}}=\beta_{+}=-0.6$ for the positrons and $\beta_{\mathrm{z}}=\beta_{-}=+0.6$ for the electrons, but the net velocity is close to zero if both fluids are combined. Overall, the bulk Lorentz factor is only close to unity in this simulation (see Fig. 12), which demonstrates that the anisotropic particle distributions is not related to the relativistic Doppler beaming. This being said, according to Lyubarsky (2005), the bulk Lorentz factor of the outflow in relativistic Petscheck-like reconnection should scale as $\gamma_{\text {out }} \sim \sqrt{\sigma}$. Indeed, it is hard to envision a scenario of fast reconnection (in the high $\sigma$ regime) where the outflowing material is not in relativistic bulk motion. PIC simulation runs that follow the evolution of the current sheet on a longer time scale typically find that the $\gamma_{\text {out }} \sim \sqrt{\sigma}$ scaling works in the high- $\sigma$ regime (Sironi and Spitkovsky 2014. K. Nalewajko 2013, private communication).

\section{Astrophysical applications}

3.1 Pulsars and pulsar wind nebulae

Pulsars are often regarded as one of the most suitable astrophysical environment for relativistic pair plasma reconnection. These objects are known to 
generate extremely magnetized plasma of pairs within their co-rotating magnetosphere. The plasma is released in the form of a relativistic magnetized wind beyond the light-cylinder surface, which is defined where the co-rotating velocity with the star equals the speed of light. In the wind region, the magnetic field lines open up and become mostly toroidal due to the fast rotation of the neutron star. This configuration naturally results in the formation of an equatorial current sheet (or "striped wind") that separates the two magnetic polarities. This is the relativistic analog of the well-known ballerina's skirt shaped heliospheric current sheet.

Reconnection in the equatorial current sheet was first proposed by Coroniti (1990) and Michel (1994) as a remedy to the "sigma-problem", i.e., to explain the transition between a Poynting-flux dominated flow formed close to the neutron star $(\sigma \gg 1)$ to the observed low- $\sigma$ pulsar wind nebulae. However, Lyubarsky and Kirk (2001) noticed that the dissipation of the current sheet would be followed by the acceleration of the wind. In the Crab pulsar, the wind would reach the termination shock before reconnection could proceed, unless the pulsar injects pairs at a higher rate than usually expected (Kirk and Skjæraasen 2003). As an alternative to the classical magnetospheric models (e.g., polar-cap, outer-gap, slot-gap), Lyubarskii (1996) suggested that reconnection in the striped wind could also explain the high-energy gammaray emission observed in pulsars (Kirk et al. 2002, Pétri 2012; Arka and Dubus 2013; Uzdensky and Spitkovsky 2014).

If, however, reconnection is inefficient in the wind zone, the striped wind is forced to dissipate at the termination shock (Lyubarsky 2003). Using particlein-cell simulations, Pétri and Lyubarsky (2007) in 1D and Sironi and Spitkovsky (2011a) in 2D and 3D showed that shock-driven reconnection is able to annihilate the magnetic structure and efficiently accelerates particles regardless of the wind properties for large magnetizations. Whether the dissipation occurs in the wind or at the termination shock, it solves only partially the sigmaproblem because the striped wind covers only a fraction of the solid angle set by the inclination angle between the rotation axis and the magnetic axis. Hence, the wind and the nebula should remain magnetically dominated at high latitudes (except for an orthogonal rotator). But, as we know from observations, pulsar wind nebulae are particle kinetic energy dominated flows, so there must be an extra mechanism to dissipate the remaining Poynting flux. Lyubarskij (1992) and Begelman (1998) argued that pulsar wind nebulae should be subject to non-axisymmetric kink-like instabilities. Their hypothesis was recently corroborated by $3 \mathrm{D}$ relativistic MHD simulations by Mizuno et al. (2011) and Porth et al. (2013, 2014). The dissipation of the magnetic energy could be done during the non-linear development of these instabilities via non-ideal MHD effects such as magnetic reconnection.

The surprising discovery of short-lived, bright gamma-ray flares from the Crab Nebula (Tavani et al. 2011; Abdo et al. 2011) could be the direct evidence of magnetic reconnection in the Nebula (Uzdensky et al. 2011; Clausen-Brown and Lyutikov 2012, Cerutti et al. 2014a). Using 2D and 3D PIC simulations, Cerutti et al. (2013, 2014b) showed that most of the features of the flares can 

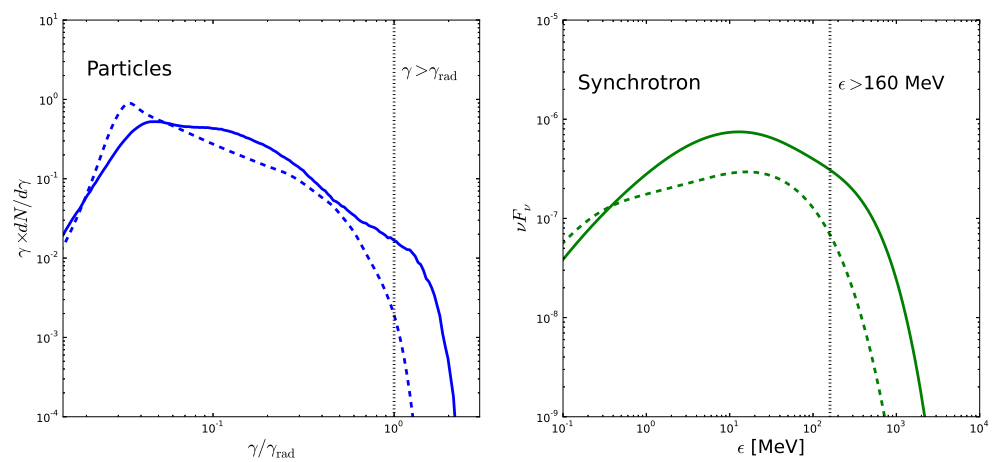

Fig. 13 Isotropically-averaged particle spectrum $(\gamma d \mathrm{~N} / d \gamma$, left panel) and synchrotron radiation energy distribution $\left(\nu F_{\nu}\right.$, right panel) in a $2 \mathrm{D}$ (solid line) and 3D (dashed line) PIC simulations of relativistic reconnection, including the effect of the radiation reaction force on the particles. The vertical dotted lines show the radiation-reaction limited energy of a particle if $E=B_{0}\left(\gamma=\gamma_{\mathrm{rad}}\right.$, left), and the corresponding maximum synchrotron photon energy $\left(\epsilon=160 \mathrm{MeV}\right.$ independent of $E$ and $B_{0}$, right). Figure adapted from Cerutti et al. (2014a).

be explained with relativistic reconnection (timescale, energetics, particle and photon spectra). In particular, these studies demonstrated that reconnection can accelerate particles above the synchrotron radiation burn-off limit Guilbert et al. 1983: de Jager et al. 1996) deep inside the reconnection layer where the electric field overcome the magnetic field (see Fig. 13), as anticipated by Kirk (2004) and Contopoulos (2007) (Sect. 1.2). This result is crucial because it can explain the emission of $>100 \mathrm{MeV}$ synchrotron radiation emitted during every Crab flare, which would be impossible to achieve in ideal MHD. The reconnection scenario would work best in the most magnetized regions of the nebula, i.e., near the poles and possibly in the jets (Cerutti et al. 2012a Lyubarsky 2012; Komissarov 2013 Mignone et al. 2013). Unfortunately, the current gamma-ray telescopes do not have the angular resolution to pin down the precise location of the flares within the Nebula.

\subsection{Jets from Active Galactic Nucleii}

Jets from active-galactic nuclei have been monitored for decades at practically all accessible electromagnetic wavelengths resulting in a very rich phenomenology (Urry and Padovani 1995). When the jet is pointing close to our line of sight, it is referred to as a "blazar". Recent observational progress in the blazar field has been immense. In particular, Cherenkov telescopes can now detect minute timescale variability in an increasing number of blazars Aharonian et al. 2007). These novel results strongly constrain the hydrodynamical models for the jet emission. 
A broader consensus has emerged regarding the qualitative nature of the "central engine". The energy source in this view is a spinning black hole or the inner accretion disk threaded by a strong magnetic field (see, e.g., Blandford and Payne 1982). This field transfers rotation energy outward as a Poynting flux. While part of the magnetic energy is used for the bulk acceleration of the jet, much of the energy remains in the magnetic field (Lyubarsky 2010) and is available to power the jet emission through dissipation by instabilities and magnetic reconnection (Giannios et al. 2009). In this picture, the jet is expected to be magnetically dominated in the emitting region, i.e., one deals with relativistic reconnection. In the following we show that applying our current understanding of relativistic reconnection to the physical conditions expected is blazar jets, reconnection can account for the extreme energetics and timescales inferred by blazar observations (for a similar approach to the modeling of the emission from gamma-ray bursts see Giannios and Spruit|2005.

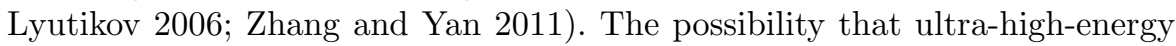
cosmic ray acceleration takes place at the current sheets of the reconnection regions of powerful jets is investigated in Giannios (2010).

The magnetic reconnection model for blazar emission: Blazar emission varies on timescales typically ranging from hours to years and is thought to reflect, in part, variations of the gas properties in the black-hole vicinity ${ }^{4}$. The recently discovered ultra-fast TeV flares from several blazar:5 (see, e.g., Aharonian et al. 2007; Albert et al. 2007) are strongly challenging the models for the blazar emission (Ghisellini and Tavecchio 2008: Giannios et al. 2009). This rare but generic blazar activity has several very revealing properties. (i) Fast flares have $\sim 10$ minute variability timescale, i.e, a factor $\sim 100$ shorter than the light-crossing time of the size of the black hole, pointing to extremely compact emitting regions. (ii) The emitting material must move with $\Gamma_{\mathrm{em}} \gtrsim 50-100$ for the $\mathrm{TeV}$ radiation to avoid anihhilation by soft radiation fields at the source (Begelman et al. 2008, Finke et al. 2008); these values of $\Gamma_{\mathrm{em}}$ are much larger than the bulk jet motion $\Gamma_{j} \sim 10$ typically inferred in blazars from radio observations (see Lister et al. 2009). (iii) For $\gtrsim 100 \mathrm{GeV}$ photons to escape the observed broad line region of the blazar PKS 1222-216, the emitting region must be located at scales $\gtrsim 0.5$ pc (Tavecchio et al. 2011). (iv) Simultaneous $\mathrm{TeV}$ and $\mathrm{GeV}$ (Fermi-LAT) observations indicate that the TeV flaring takes place on top of longer day-long blazar activity (e.g. Tanaka et al. 2011). (v) Fast flares may come in a repetitive fashion of similar events as observed in PKS 2155-304 (Aharonian et al. 2007). Taken together, these inferences are extremely constraining for the models for the blazar emission.

Giannios et al. (2009) argued that the ultra-fast variability must be generated internally in the jet by MHD instabilities. In strongly magnetized jets, the reconnection process injects energetic particles in compact, fast moving regions. These regions are natural emitters of powerful flares. Furthermore, the

\footnotetext{
${ }^{4}$ Several hours is the event-horizon light-crossing time of a billion solar-mass black holemass typically inferred for the central engine in blazars: $t_{\text {cross }}=2 G M_{\mathrm{BH}} / c^{3} \simeq 10^{4} M_{9} \mathrm{~s}$.

5 Including Mrk 421, Mrk 501, PKS 2155-304, PKS 1222-216, and BL Lac.
} 
emitting material is expected to be faster than the jet on average allowing for $\mathrm{TeVs}$ to escape the source. For a jet moving with bulk $\Gamma_{\mathrm{j}} \sim 10-20$ and a plasmoid being ejected with bulk $\gamma_{\text {out }} \simeq \sqrt{\sigma}$ (as measured in the rest frame of the jet), the emitting region moves with $\Gamma_{\mathrm{em}} \simeq 2 \Gamma_{\mathrm{j}} \gamma_{\text {out }}$ (in the frame of the host galaxy). For $\sigma \sim$ several, one can easily account for the required $\Gamma_{\mathrm{em}} \gtrsim 50$. Applications of the model to fit spectra of specific sources are reported in Giannios et al. (2010); Nalewajko et al. (2011).

The Giannios et al. (2009) model is based on a simplified picture for the reconnection geometry adopting a steady state reconnection model. As pointed out by Narayan and Piran (2012) steady reconnection cannot account for the fastest evolving blazar flares because the variability timescale is limited by the reconnection speed $\beta_{\text {in }}<1$. However, assuming steady reconnection is oversimplistic. Solar and Earth magnetosphere observations and recent advances in theory and numerical simulations (see previous Sections) have revealed that reconnection is an inherently time-dependent, highly dynamic, process (see, e.g., Lin et al. 2005: Park et al. 2006; Karlický and Kliem 2010). These timedependent aspects of reconnection are crucial in understanding the fastest timescales involved in blazar flaring. For the physical conditions prevailing in jets, the reconnection current sheets are expected to suffer from tearing instabilities that lead to their fragmentation to a large number of plasmoids (Loureiro et al. 2007, Bhattacharjee et al. 2009). The plasmoids grow rapidly through mergers before leaving the reconnection region. Occasionally plasmoids undergo significant growth to a scale of order of that of the reconnection region, forming "monster" plasmoids (Uzdensky et al. 2010, see Fig. 14; left panel). The relativistic motion of the plasmoids in the rest frame of the jet results in additional beaming of their emission (i.e., beyond that induced by the jet motion). When the layer's orientation is such that plasmoids beam their emission towards the observer, powerful and fast evolving flares emerge. Here we focus on the characteristic observed timescales and luminosities resulting from plasmoids that form in the reconnection region. For simplicity, we assume that the dissipated energy is efficiently converted into radiation ${ }^{6}$

Giannios (2013) demonstrated that a broad range of blazar phenomenology can be qualitatively understood in the context of plasmoid-dominated reconnection. The virtue of the model is that it can be applied to all blazar sources with observed fast flaring for similar adopted parameters. The model favors pc-scale dissipation for the origin of the fast flaring and provides theoretical motivation for such dissipation distance. Another interesting aspect of the model is that a sequence of fast flares is expected to have similar timescale set by the size of the reconnection layer as observed in PKS 2155. This work has demonstrated that the tight energetic, emitter Lorentz factor, and timescale constraints (i)-(v) are satisfied in the reconnection model. More importantly, the basic assumptions of the Giannios 2013 analysis on the properties of the

\footnotetext{
6 In practice the blazar emission is likely to result of ultrarelativistic electrons cooling via synchrotron radiation and Compton scattering. As discussed in previous sections, relativistic reconnection is an effective means of accelerating particles to such extreme energies.
} 

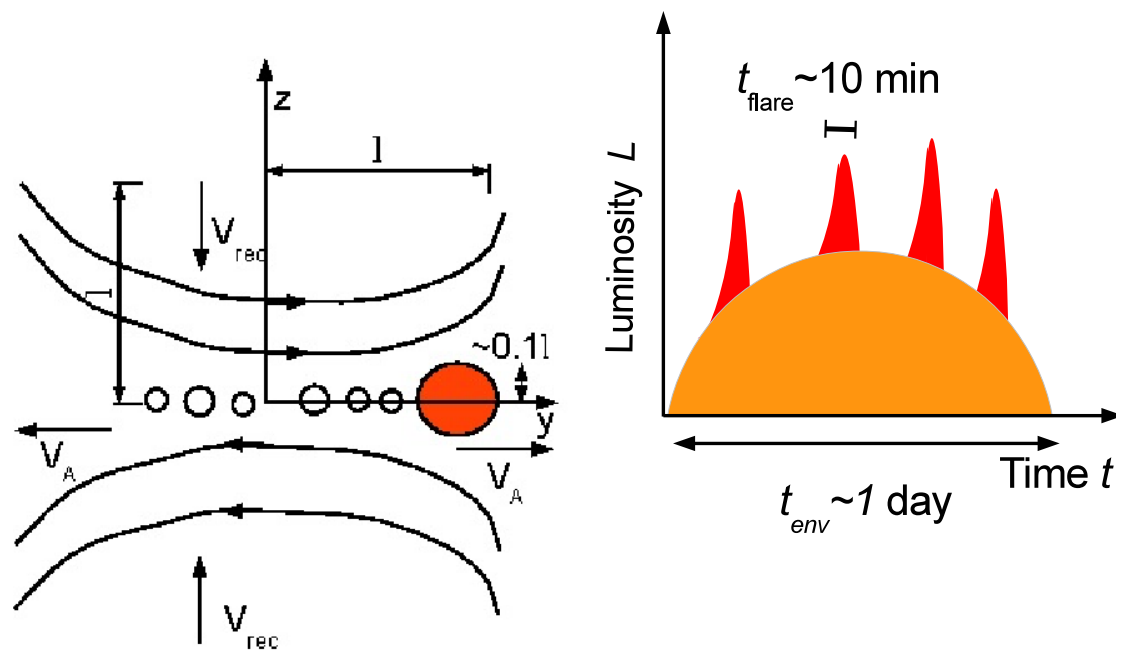

Fig. 14 Left Panel: Schematic representation of the geometry of reconnection process shown in a frame comoving with the jet. Magnetic field lines of opposite polarity annihilate at the $x-y$ plane with speed $v_{\text {rec }}=\beta_{\text {in }} c$. The reconnection layer fragments to a large number of plasmoids. Regularily, plasmoids undergo multiple mergers resulting in a "monster" plasmoid (shaded blob). Right Panel: Sketch of the emission from plasmoid-dominated reconnection. The reconnection proceeds on a global timescale $t_{\mathrm{rec}}=l / \beta_{\mathrm{in}} c$, powering $\sim 1$ day long flares (or envelope emission). Regularily, plasmoids grow to become "monster" plasmoids (shaded blob) giving rise to powerful, fast-evolving flares of duration $t_{\text {flare }} \sim 10$ minutes. Several fast flares are expected from a single reconnection event.

reconnection layer have been fully verified by PIC simulations since then (see previous Sections).

In the following of this Section we make a plausibility argument for the model: we estimate the characteristic observed timescales and luminosities resulting from plasmoids that form in the reconnection region (for full derivations see Giannios 2013). To this end, we consider a blob (or plasmoid) emerging from the reconnection layer moving with the Alfvén speed of the reconnection upstream, i.e, with a corresponding bulk Lorentz factor $\gamma_{\text {out }} \simeq \sqrt{\sigma}$ (measured in the jet rest frame) and of size $R_{\mathrm{p}}^{\prime \prime}=f l^{\prime}$, where $l^{\prime}$ is the characteristic scale of the reconnection region and $f$ is a dimensionless parameter of the order of 0.1 , as expected for the largest, "monster" plasmoids (Uzdensky et al. 2010); hereafter, primed (double primed) quantities are measured in the rest frame of the jet (emitting blob) 7 The observed characteristic variability time for the plasmoid emission is $t_{\mathrm{v}} \simeq R_{\mathrm{p}}^{\prime \prime} / \delta_{p} c$, where $\delta_{\mathrm{p}}$ is the Doppler boost of the

7 We assume that the plasmoid instability operates across the whole length of the current sheet, as opposed to a situation where central, very compact, dissipation region forms and is 
plasmoid radiation towards the observer. For a central engine in which the magnetic field varies on a dynamical time $\sim R_{\mathrm{Sch}} / c$, the characteristic scale of the reconnection region can be estimated to be $l^{\prime} \simeq \Gamma_{\mathrm{j}} R_{\mathrm{Sch}}$ resulting in

$$
t_{\mathrm{v}}=\frac{f \Gamma_{j} R_{\mathrm{Sch}}}{\delta_{\mathrm{p}} c}=400 f_{-1} \Gamma_{\mathrm{j}, 20} M_{9} \delta_{p, 50}^{-1} \quad \mathrm{~s},
$$

where $\delta_{\mathrm{p}}=50 \delta_{\mathrm{p}, 50}, f=0.1 f_{-1}, \Gamma_{\mathrm{j}}=20 \Gamma_{\mathrm{j}, 20} . f \sim 0.1$ describes the largest plasmoids expected in the layer (Uzdensky et al. 2010). Flaring on several minute timescale is therefore expected in this picture.

Consider a jet emerging from a supermassive black hole with (isotropic equivalent) power $L_{\text {iso }}$, opening angle $\theta_{j}$ and Lorentz factor $\Gamma_{j}$. We also assume that $\theta_{j} \Gamma_{j}=0.2$ as indicated by observations (Pushkarev et al. 2009). The typical bulk Lorentz factor of gamma-ray active blazars is $\Gamma_{j} \sim 10-20$ (Savolainen et al. 2010, Piner et al. 2012). The energy density at the dissipation, or "blazar", zone is

$$
U_{\mathrm{j}}^{\prime}=\frac{L_{\text {iso }}}{4 \pi\left(\theta_{\mathrm{j}} R_{\mathrm{diss}}\right)^{2} \delta_{\mathrm{j}}^{4} c} .
$$

The dissipation distance $R_{\text {diss }}$ is estimated requiring that the reconnection proceeds within the expansion time of the jet $\left(R_{\mathrm{diss}} / \Gamma_{j} c \sim l^{\prime} / \epsilon c\right)$.

Pressure balance across the reconnection layer requires the energy density of the plasmoid to be similar to that of the jet $U_{p}^{\prime \prime} \sim U_{j}^{\prime}$. Assuming efficient conversion of dissipated energy into radiation, the rest-frame luminosity of the plasmoid is thus $L_{\mathrm{p}, \mathrm{obs}}=\delta_{p}^{4} L^{\prime \prime}=\delta_{p}^{4} U_{\mathrm{p}}^{\prime \prime} 4 \pi R_{\mathrm{p}}^{\prime \prime 2} c$. Putting everything together, the observed luminosity of the plasmoid is (Giannios 2013)

$$
L_{\mathrm{p}, \mathrm{obs}}=10^{47} \frac{\beta_{\mathrm{in},-1}^{2} f_{-1}^{2} \delta_{p, 50}^{4} L_{\mathrm{iso}, 48}}{\delta_{j, 20}^{4}} \mathrm{erg} / \mathrm{s} .
$$

The Doppler factor of the plasmoid $\delta_{\mathrm{p}}$ depends on several parameters. It is related to $\Gamma_{j}, \gamma_{\text {out }}$, the angle of the plasmoid with respect to the jet motion and the observer's angle of sight. For typical situations where the reconnection layer is at a large $\theta \sim \pi / 2$ angle with respect to the jet propagation (as seen in the jet rest) and fairly aligned with the observer (giving powerful flares) $\delta_{\mathrm{p}} \sim \Gamma_{\mathrm{j}} \gamma_{\text {out }}$. One can see (see Eq. 17) that powerful flares on a timescale of $\sim 10 \mathrm{~min}$ is possible even with very modest relativistic motions within the jet $\gamma_{\text {out }} \sim 2$.

Ejection of multiple monster plasmoids: During a reconnection event multiple monster plasmoids are expected to form. 2D simulations (Loureiro et al. 2012) indicate that monster plasmoids form every few Alfvén times $t_{A}$ or at a rate of $\sim 0.3 t_{A}^{-1}$. It appears likely that $2 \mathrm{D}$ simulations underestimate the rate of formation of monster plasmoids. The actual rate may be higher when the 3D

surrounded by extended magnetic separatrices (the slow shocks in Petscheck model) across which most of the plasma flows. In the latter case, the monster plasmoids may be smaller. 
structure of the layer is considered (Sironi and Spitkovsky 2014). If monster plasmoids emerge at a rate $\sim(0.3-3) t_{A}^{-1}$, some $(3-30) / \beta_{\text {in, }-1}$ plasmoids are expected from a single reconnection layer powering multiple flares. A sketch of such pattern is shown in Fig. 14 .

The "envelope emission" from the reconnection region: The bulk motion of a monster plasmoid is expected to be similar to the speed of other structures (e.g. smaller plasmoids) leaving the reconnection region. When the plasmoid emission is beamed towards the observer (powering a fast flare), the overall emission from the current layer is also beamed by a similar factor. The emission from the layer forms a slower-evolving "envelope". In the following we estimate the timescale and luminosity of the emission from the reconnection layer.

At the dissipation distance $R_{\text {diss }}$, the reconnection proceeds within the expansion time of the jet $\left(R_{\text {diss }} / \Gamma_{j} c \sim l^{\prime} / \beta_{\text {in }} c\right)$ which is observed to last for $t_{\mathrm{exp}, \mathrm{obs}} \simeq R_{\mathrm{diss}} / \Gamma_{j}^{2} c$. Therefore, $t_{\mathrm{exp}, \mathrm{obs}}$ corresponds to the observed duration of the envelope emission which is simply (using also Eq. (15)):

$$
t_{\mathrm{env}}=\frac{R_{\mathrm{diss}}}{\Gamma_{j}^{2} c}=10^{5} \frac{M_{9}}{\beta_{\mathrm{in},-1}} \quad \mathrm{~s} .
$$

The duration of the envelope emission is $\sim$ days. Such timescale is characteristic of blazar flares.

The (lab frame) energy available to power the envelope emission is $E_{\text {env }}=$ $U_{\mathrm{j}} 2 l^{\prime 3} / \Gamma_{\mathrm{j}}$, where $U_{j}=\Gamma_{j}^{2} U_{j}^{\prime}$ is the energy density of the jet and $2 l^{\prime 3} / \Gamma_{\mathrm{j}}$ accounts for (lab frame) volume of the reconnection region that powers each minijet (see Fig. 14. The emitted luminosity of the reconnection region is $E_{\text {env }} / t_{\text {env }}$. It can be converted into observed luminosity by accounting for beaming factor of the emission $\sim \delta_{p}^{2}$ :

$$
L_{\mathrm{env}, \mathrm{obs}} \simeq 2 \Gamma_{\mathrm{j}}^{2} \delta_{\mathrm{p}}^{2} l^{\prime 2} U_{\mathrm{j}}^{\prime} \beta_{\mathrm{in}} c=3 \times 10^{46} \frac{\Gamma_{\mathrm{j}, 20}^{2} \delta_{\mathrm{p}, 50}^{2} \beta_{\mathrm{in},-1}^{3} L_{\mathrm{iso}, 48}}{\delta_{j, 20}^{4}} \mathrm{erg} / \mathrm{s} .
$$

The envelope emission is quite bright. Dividing Eqs. (17) and (19), one arrives to a fairly simple expression for the ratio of the plasmoid to envelope luminosities $L_{p} / L_{\mathrm{env}} \sim 3 f_{-1}^{2} \delta_{\mathrm{p}, 50}^{2} /\left(\Gamma_{\mathrm{j}, 20}^{2} \beta_{\mathrm{in},-1}\right)$. The luminosity contrast depends only on the Lorentz factor of the minijet in the rest frame of the jet $\gamma_{\mathrm{p}} \simeq \delta_{\mathrm{p}} / \Gamma_{\mathrm{j}}$, the size of the plasmoid parametrized by $f$, and the reconnection sped $\beta_{\text {in }}$. The observed luminosity ratio is of order unity constraining $\delta_{\mathrm{p}, 50} / \Gamma_{\mathrm{j}, 20} \sim 1$ for $\beta_{\text {in }} \sim f \sim 0.1$. The ratio $\delta_{\mathrm{p}, 50} / \Gamma_{\mathrm{j}, 20}$ is determined by the reconnection-induced bulk motions in the jet and points to $\gamma_{\text {out }} \sim 2$ or, equivalently, moderately magnetized jet with $\sigma \sim$ several.

Most of the current numerical work on relativistic reconnection (and this review so far) has focused on the case of electron-positron plasmas. The composition of the jet flow is still an open question but an electron-proton jet is a strong possibility. Electron-ion reconnection is more challenging, on a numerical level, than electron-positron reconnection, since the computation has to resolve the small scales of electrons, yet the system evolves on the longer 
ion timescales. However, the physics of relativistic electron-proton reconnection, yet still at an early stage of investigation, shows remarkable similarities with electron-positron reconnection (e.g., Melzani et al. 2014). A detailed investigation of relativistic reconnection in the case of unequal mass charges is of paramount importance to obtaining predictions for the acceleration of electrons and cosmic rays in blazar jets.

\section{Conclusion}

There has been significant progress in our understanding of relativistic reconnection in recent years, thanks to both analytical works and numerical simulations. One important outcome is that plasma instabilities in current sheets play a crucial role in the dynamics of reconnection. In particular, the tearing instability which fragments the current sheet, leads to fast reconnection and efficient non-thermal particle acceleration. Particle-in-cell simulations are now large enough to unambiguously identify broad, hard power laws in the particle energy distributions (in the high-magnetization limit). The power-law index is typically harder than the universal $\sim-2$ index expected in shock acceleration. These impressive developments were also motivated by puzzling observations of high-energy phenomena in the Universe, especially flaring gamma-ray sources. Ultra-rapid gamma-ray flares discovered in the Crab Nebula and in several AGN jets are too fast and too bright to be explained by conventional models. Particle beaming and relativistic bulk motions associated with relativistic reconnection can alleviate these difficulties. We expect fast new developments in this field, with more applications to astrophysical objects.

Acknowledgements We thank the referees for useful comments that helped to improve the manuscript. LS is supported by NASA through Einstein Postdoctoral Fellowship grant number PF1-120090 awarded by the Chandra X-ray Center, which is operated by the Smithsonian Astrophysical Observatory for NASA under contract NAS8-03060. BC acknowledges support from the Lyman Spitzer Jr. Fellowship awarded by the Department of Astrophysical Sciences at Princeton University, and the Max-Planck/Princeton Center for Plasma Physics. DG acknowledges support from the NASA grant NNX13AP13G.

\section{References}

A.A. Abdo, M. Ackermann, M. Ajello, A. Allafort, L. Baldini, J. Ballet, G. Barbiellini, D. Bastieri, K. Bechtol, R. Bellazzini, B. Berenji, R.D. Blandford, E.D. Bloom, E. Bonamente, A.W. Borgland, A. Bouvier, T.J. Brandt, J. Bregeon, A. Brez, M. Brigida, P. Bruel, R. Buehler, S. Buson, G.A. Caliandro, R.A. Cameron, A. Cannon, P.A. Caraveo, J.M. Casandjian, Ö. Çelik, E. Charles, A. Chekhtman, C.C. Cheung, J. Chiang, S. Ciprini, R. Claus, J. Cohen-Tanugi, L. Costamante, S. Cutini, F. D'Ammando, C.D. Dermer, A. de Angelis, A. de Luca, F. de Palma, S.W. Digel, E. do Couto e Silva, P.S. Drell, A. Drlica-Wagner, R. Dubois, D. Dumora, C. Favuzzi, S.J. Fegan, E.C. Ferrara, W.B. Focke, P. Fortin, M. Frailis, Y. Fukazawa, S. Funk, P. Fusco, F. Gargano, D. Gasparrini, N. Gehrels, S. Germani, N. Giglietto, F. Giordano, M. Giroletti, T. Glanzman, G. Godfrey, I.A. Grenier, M.-H. Grondin, J.E. Grove, S. Guiriec, D. Hadasch, Y. Hanabata, A.K. Harding, K. Hayashi, M. Hayashida, E. Hays, D. Horan, R. Itoh, 
G. Jóhannesson, A.S. Johnson, T.J. Johnson, D. Khangulyan, T. Kamae, H. Katagiri, J. Kataoka, M. Kerr, J. Knödlseder, M. Kuss, J. Lande, L. Latronico, S.-H. Lee, M. Lemoine-Goumard, F. Longo, F. Loparco, P. Lubrano, G.M. Madejski, A. Makeev, M. Marelli, M.N. Mazziotta, J.E. McEnery, P.F. Michelson, W. Mitthumsiri, T. Mizuno, A.A. Moiseev, C. Monte, M.E. Monzani, A. Morselli, I.V. Moskalenko, S. Murgia, T. Nakamori, M. Naumann-Godo, P.L. Nolan, J.P. Norris, E. Nuss, T. Ohsugi, A. Okumura, N. Omodei, J.F. Ormes, M. Ozaki, D. Paneque, D. Parent, V. Pelassa, M. Pepe, M. Pesce-Rollins, M. Pierbattista, F. Piron, T.A. Porter, S. Rainò, R. Rando, P.S. Ray, M. Razzano, A. Reimer, O. Reimer, T. Reposeur, S. Ritz, R.W. Romani, H.F.-W. Sadrozinski, D. Sanchez, P.M.S. Parkinson, J.D. Scargle, T.L. Schalk, C. Sgrò, E.J. Siskind, P.D. Smith, G. Spandre, P. Spinelli, M.S. Strickman, D.J. Suson, H. Takahashi, T. Takahashi, T. Tanaka, J.B. Thayer, D.J. Thompson, L. Tibaldo, D.F. Torres, G. Tosti, A. Tramacere, E. Troja, Y. Uchiyama, J. Vandenbroucke, V. Vasileiou, G. Vianello, V. Vitale, P. Wang, K.S. Wood, Z. Yang, M. Ziegler, Gamma-Ray Flares from the Crab Nebula. Science 331, 739 (2011). doi:10.1126/science.1199705

F. Aharonian, A.G. Akhperjanian, A.R. Bazer-Bachi, B. Behera, M. Beilicke, W. Benbow, D. Berge, K. Bernlöhr, C. Boisson, O. Bolz, V. Borrel, T. Boutelier, I. Braun, E. Brion, A.M. Brown, R. Bühler, I. Büsching, T. Bulik, S. Carrigan, P.M. Chadwick, A.C. Clapson, L.-M. Chounet, G. Coignet, R. Cornils, L. Costamante, B. Degrange, H.J. Dickinson, A. Djannati-Ataï, W. Domainko, L.O. Drury, G. Dubus, J. Dyks, K. Egberts, D. Emmanoulopoulos, P. Espigat, C. Farnier, F. Feinstein, A. Fiasson, A. Förster, G. Fontaine, S. Funk, S. Funk, M. Füßling, Y.A. Gallant, B. Giebels, J.F. Glicenstein, B. Glück, P. Goret, C. Hadjichristidis, D. Hauser, M. Hauser, G. Heinzelmann, G. Henri, G. Hermann, J.A. Hinton, A. Hoffmann, W. Hofmann, M. Holleran, S. Hoppe, D. Horns, A. Jacholkowska, O.C. de Jager, E. Kendziorra, M. Kerschhaggl, B. Khélifi, N. Komin, K. Kosack, G. Lamanna, I.J. Latham, R. Le Gallou, A. Lemière, M. Lemoine-Goumard, J.-P. Lenain, T. Lohse, J.M. Martin, O. Martineau-Huynh, A. Marcowith, C. Masterson, G. Maurin, T.J.L. McComb, R. Moderski, E. Moulin, M. de Naurois, D. Nedbal, S.J. Nolan, J.-P. Olive, K.J. Orford, J.L. Osborne, M. Ostrowski, M. Panter, G. Pedaletti, G. Pelletier, P.-O. Petrucci, S. Pita, G. Pühlhofer, M. Punch, S. Ranchon, B.C. Raubenheimer, M. Raue, S.M. Rayner, M. Renaud, J. Ripken, L. Rob, L. Rolland, S. Rosier-Lees, G. Rowell, B. Rudak, J. Ruppel, V. Sahakian, A. Santangelo, L. Saugé, S. Schlenker, R. Schlickeiser, R. Schröder, U. Schwanke, S. Schwarzburg, S. Schwemmer, A. Shalchi, H. Sol, D. Spangler, Ł. Stawarz, R. Steenkamp, C. Stegmann, G. Superina, P.H. Tam, J.-P. Tavernet, R. Terrier, C. van Eldik, G. Vasileiadis, C. Venter, J.P. Vialle, P. Vincent, M. Vivier, H.J. Völk, F. Volpe, S.J. Wagner, M. Ward, A.A. Zdziarski, An Exceptional Very High Energy Gamma-Ray Flare of PKS 2155-304. Astrophys. J. Lett. 664, 71-74 (2007). doi:10.1086/520635

J. Albert, E. Aliu, H. Anderhub, P. Antoranz, A. Armada, C. Baixeras, J.A. Barrio, H. Bartko, D. Bastieri, J.K. Becker, W. Bednarek, K. Berger, C. Bigongiari, A. Biland, R.K. Bock, P. Bordas, V. Bosch-Ramon, T. Bretz, I. Britvitch, M. Camara, E. Carmona, A. Chilingarian, J.A. Coarasa, S. Commichau, J.L. Contreras, J. Cortina, M.T. Costado, V. Curtef, V. Danielyan, F. Dazzi, A. De Angelis, C. Delgado, R. de los Reyes, B. De Lotto, E. Domingo-Santamaría, D. Dorner, M. Doro, M. Errando, M. Fagiolini, D. Ferenc, E. Fernández, R. Firpo, J. Flix, M.V. Fonseca, L. Font, M. Fuchs, N. Galante, R.J. García-López, M. Garczarczyk, M. Gaug, M. Giller, F. Goebel, D. Hakobyan, M. Hayashida, T. Hengstebeck, A. Herrero, D. Höhne, J. Hose, D. Hrupec, C.C. Hsu, P. Jacon, T. Jogler, R. Kosyra, D. Kranich, R. Kritzer, A. Laille, E. Lindfors, S. Lombardi, F. Longo, J. López, M. López, E. Lorenz, P. Majumdar, G. Maneva, K. Mannheim, O. Mansutti, M. Mariotti, M. Martínez, D. Mazin, C. Merck, M. Meucci, M. Meyer, J.M. Miranda, R. Mirzoyan, S. Mizobuchi, A. Moralejo, D. Nieto, K. Nilsson, J. Ninkovic, E. Oña-Wilhelmi, N. Otte, I. Oya, D. Paneque, M. Panniello, R. Paoletti, J.M. Paredes, M. Pasanen, D. Pascoli, F. Pauss, R. Pegna, M. Persic, L. Peruzzo, A. Piccioli, E. Prandini, N. Puchades, A. Raymers, W. Rhode, M. Ribó, J. Rico, M. Rissi, A. Robert, S. Rügamer, A. Saggion, T. Saito, A. Sánchez, P. Sartori, V. Scalzotto, V. Scapin, R. Schmitt, T. Schweizer, M. Shayduk, K. Shinozaki, S.N. Shore, N. Sidro, A. Sillanpää, D. Sobczynska, A. Stamerra, L.S. Stark, L. Takalo, F. Tavecchio, P. Temnikov, D. Tescaro, M. Teshima, D.F. Torres, N. Turini, H. Vankov, V. Vitale, R.M. Wagner, T. Wibig, W. 
Wittek, F. Zandanel, R. Zanin, J. Zapatero, Variable Very High Energy $\gamma$-Ray Emission from Markarian 501. Astrophys. J. 669, 862-883 (2007). doi:10.1086/521382

I. Arka, G. Dubus, Pulsed high-energy $\gamma$-rays from thermal populations in the current sheets of pulsar winds. Astron. Astrophys. 550, 101 (2013). doi:10.1051/0004-6361/201220110

G. Baumann, Å. Nordlund, Particle-in-cell Simulation of Electron Acceleration in Solar Coronal Jets. Astrophys. J. Lett. 759, 9 (2012). doi:10.1088/2041-8205/759/1/L9

M.C. Begelman, Instability of Toroidal Magnetic Field in Jets and Plerions. Astrophys. J. 493, 291-300 (1998). doi:10.1086/305119

M.C. Begelman, A.C. Fabian, M.J. Rees, Implications of very rapid TeV variability in blazars. Mon. Not. R. Astron. Soc. 384, 19-23 (2008). doi:10.1111/j.17453933.2007.00413.x

N. Bessho, A. Bhattacharjee, Collisionless Reconnection in an Electron-Positron Plasma. Physical Review Letters 95(24), 245001 (2005). doi:10.1103/PhysRevLett.95.245001

N. Bessho, A. Bhattacharjee, Fast collisionless reconnection in electron-positron plasmas. Physics of Plasmas 14(5), 056503 (2007). doi:10.1063/1.2714020

N. Bessho, A. Bhattacharjee, Fast Magnetic Reconnection and Particle Acceleration in Relativistic Low-density Electron-Positron Plasmas without Guide Field. Astrophys. J. 750, 129 (2012). doi:10.1088/0004-637X/750/2/129

A. Bhattacharjee, Y.-M. Huang, H. Yang, B. Rogers, Fast reconnection in high-Lundquistnumber plasmas due to the plasmoid Instability. Physics of Plasmas 16(11), 112102 (2009). doi:10.1063/1.3264103

C.K. Birdsall, A.B. Langdon, Plasma Physics via Computer Simulation 1991

J. Birn, M. Hesse, Geospace Environment Modeling (GEM) magnetic reconnection challenge: Resistive tearing, anisotropic pressure and hall effects. J. Geophys. Res. 106, 3737-3750 (2001). doi:10.1029/1999JA001001

D. Biskamp, Magnetic Reconnection in Plasmas 2000

R.D. Blandford, D.G. Payne, Hydromagnetic flows from accretion discs and the production of radio jets. Mon. Not. R. Astron. Soc. 199, 883-903 (1982)

B. Cerutti, D.A. Uzdensky, M.C. Begelman, Extreme Particle Acceleration in Magnetic Reconnection Layers: Application to the Gamma-Ray Flares in the Crab Nebula. Astrophys. J. 746, 148 (2012a). doi:10.1088/0004-637X/746/2/148

B. Cerutti, G.R. Werner, D.A. Uzdensky, M.C. Begelman, Beaming and Rapid Variability of High-energy Radiation from Relativistic Pair Plasma Reconnection. Astrophys. J. Lett. 754, 33 (2012b). doi:10.1088/2041-8205/754/2/L33

B. Cerutti, G.R. Werner, D.A. Uzdensky, M.C. Begelman, Simulations of Particle Acceleration beyond the Classical Synchrotron Burnoff Limit in Magnetic Reconnection: An Explanation of the Crab Flares. Astrophys. J. 770, 147 (2013). doi:10.1088/0004$637 \mathrm{X} / 770 / 2 / 147$

B. Cerutti, G.R. Werner, D.A. Uzdensky, M.C. Begelman, Gamma-ray flares in the Crab Nebula: A case of relativistic reconnection?a). Physics of Plasmas 21(5), 056501 (2014a). doi:10.1063/1.4872024

B. Cerutti, G.R. Werner, D.A. Uzdensky, M.C. Begelman, Three-dimensional Relativistic Pair Plasma Reconnection with Radiative Feedback in the Crab Nebula. Astrophys. J. 782, 104 (2014b). doi:10.1088/0004-637X/782/2/104

L.-J. Chen, A. Bhattacharjee, P.A. Puhl-Quinn, H. Yang, N. Bessho, S. Imada, S. Mühlbachler, P.W. Daly, B. Lefebvre, Y. Khotyaintsev, A. Vaivads, A. Fazakerley, E. Georgescu, Observation of energetic electrons within magnetic islands. Nature Physics 4, 19-23 (2008). doi:10.1038/nphys777

E. Clausen-Brown, M. Lyutikov, Crab nebula gamma-ray flares as relativistic reconnection minijets. Mon. Not. R. Astron. Soc. 426, 1374-1384 (2012). doi:10.1111/j.13652966.2012.21349.x

L. Comisso, F.A. Asenjo, Thermal-Inertial Effects on Magnetic Reconnection in Relativistic Pair Plasmas. Physical Review Letters 113(4), 045001 (2014). doi:10.1103/PhysRevLett.113.045001

I. Contopoulos, The magnetic field topology in the reconnecting pulsar magnetosphere. Astron. Astrophys. 472, 219-223 (2007). doi:10.1051/0004-6361:20077167

F.V. Coroniti, Magnetically striped relativistic magnetohydrodynamic winds - The Crab Nebula revisited. Astrophys. J. 349, 538-545 (1990). doi:10.1086/168340 
W. Daughton, H. Karimabadi, Collisionless magnetic reconnection in large-scale electronpositron plasmas. Physics of Plasmas 14(7), 072303 (2007). doi:10.1063/1.2749494

O.C. de Jager, A.K. Harding, P.F. Michelson, H.I. Nel, P.L. Nolan, P. Sreekumar, D.J. Thompson, Gamma-Ray Observations of the Crab Nebula: A Study of the SynchroCompton Spectrum. Astrophys. J. 457, 253 (1996). doi:10.1086/176726

J.F. Drake, M. Swisdak, H. Che, M.A. Shay, Electron acceleration from contracting magnetic islands during reconnection. Nature 443, 553-556 (2006). doi:10.1038/nature05116

J.F. Drake, M. Opher, M. Swisdak, J.N. Chamoun, A Magnetic Reconnection Mechanism for the Generation of Anomalous Cosmic Rays. Astrophys. J. 709, 963-974 (2010). doi:10.1088/0004-637X/709/2/963

J.D. Finke, C.D. Dermer, M. Böttcher, Synchrotron Self-Compton Analysis of TeV X-RaySelected BL Lacertae Objects. Astrophys. J. 686, 181-194 (2008). doi:10.1086/590900

G. Ghisellini, F. Tavecchio, Rapid variability in TeV blazars: the case of PKS2155-304. Mon. Not. R. Astron. Soc. 386, 28-32 (2008). doi:10.1111/j.1745-3933.2008.00454.x

D. Giannios, UHECRs from magnetic reconnection in relativistic jets. Mon. Not. R. Astron. Soc. 408, 46-50 (2010). doi:10.1111/j.1745-3933.2010.00925.x

D. Giannios, Reconnection-driven plasmoids in blazars: fast flares on a slow envelope. Mon. Not. R. Astron. Soc. 431, 355-363 (2013). doi:10.1093/mnras/stt167

D. Giannios, H.C. Spruit, Spectra of Poynting-flux powered GRB outflows. Astron. Astrophys. 430, 1-7 (2005). doi:10.1051/0004-6361:20047033

D. Giannios, D.A. Uzdensky, M.C. Begelman, Fast TeV variability in blazars: jets in a jet. Mon. Not. R. Astron. Soc. 395, 29-33 (2009). doi:10.1111/j.1745-3933.2009.00635.x

D. Giannios, D.A. Uzdensky, M.C. Begelman, Fast TeV variability from misaligned minijets in the jet of M87. Mon. Not. R. Astron. Soc. 402, 1649-1656 (2010). doi:10.1111/j.13652966.2009.16045.x

J. Goodman, D. Uzdensky, Reconnection in Marginally Collisionless Accretion Disk Coronae. Astrophys. J. 688, 555-558 (2008). doi:10.1086/592345

J. Graf von der Pahlen, D. Tsiklauri, The effect of guide-field and boundary conditions on collisionless magnetic reconnection in a stressed X-point collapse. Physics of Plasmas 21(1), 012901 (2014). doi:10.1063/1.4861258

P.W. Guilbert, A.C. Fabian, M.J. Rees, Spectral and variability constraints on compact sources. Mon. Not. R. Astron. Soc. 205, 593-603 (1983)

F. Guo, H. Li, W. Daughton, Y.-H. Liu, Formation of Hard Power-laws in the Energetic Particle Spectra Resulting from Relativistic Magnetic Reconnection. ArXiv e-prints (2014)

E.G. Harris Nuevo Cimento 23, 115 (1962)

K. Higashimori, M. Hoshino, The relation between ion temperature anisotropy and formation of slow shocks in collisionless magnetic reconnection. Journal of Geophysical Research (Space Physics) 117, 1220 (2012). doi:10.1029/2011JA016817

Y.-M. Huang, A. Bhattacharjee, Scaling laws of resistive magnetohydrodynamic reconnection in the high-Lundquist-number, plasmoid-unstable regime. Physics of Plasmas 17(6), 062104 (2010). doi:10.1063/1.3420208

C.H. Jaroschek, H. Lesch, R.A. Treumann, Relativistic Kinetic Reconnection as the Possible Source Mechanism for High Variability and Flat Spectra in Extragalactic Radio Sources. Astrophys. J. Lett. 605, 9-12 (2004). doi:10.1086/420767

C.H. Jaroschek, M. Hoshino, H. Lesch, R.A. Treumann, Stochastic particle acceleration by the forced interaction of relativistic current sheets. Advances in Space Research 41, 481-490 (2008). doi:10.1016/j.asr.2007.07.001

H. Ji, W. Daughton, Phase diagram for magnetic reconnection in heliophysical, astrophysical, and laboratory plasmas. Physics of Plasmas 18(11), 111207 (2011). doi:10.1063/1.3647505

D. Kagan, M. Milosavljević, A. Spitkovsky, A Flux Rope Network and Particle Acceleration in Three-dimensional Relativistic Magnetic Reconnection. Astrophys. J. 774, 41 (2013). doi:10.1088/0004-637X/774/1/41

M. Karlický, B. Kliem, Reconnection of a Kinking Flux Rope Triggering the Ejection of a Microwave and Hard X-ray Source I. Observations and Interpretation. Solar Phys. 266, 71-89 (2010). doi:10.1007/s11207-010-9606-4

J.G. Kirk, Particle Acceleration in Relativistic Current Sheets. Physical Review Letters 92(18), 181101 (2004). doi:10.1103/PhysRevLett.92.181101 
J.G. Kirk, O. Skjæraasen, Dissipation in Poynting-Flux-dominated Flows: The $\sigma$-Problem of the Crab Pulsar Wind. Astrophys. J. 591, 366-379 (2003). doi:10.1086/375215

J.G. Kirk, O. Skjæraasen, Y.A. Gallant, Pulsed radiation from neutron star winds. Astron. Astrophys. 388, 29-32 (2002). doi:10.1051/0004-6361:20020599

S.S. Komissarov, Magnetic dissipation in the Crab nebula. Mon. Not. R. Astron. Soc. 428, 2459-2466 (2013). doi:10.1093/mnras/sts214

D.A. Larrabee, R.V.E. Lovelace, M.M. Romanova, Lepton Acceleration by Relativistic Collisionless Magnetic Reconnection. Astrophys. J. 586, 72-78 (2003). doi:10.1086/367640

J. Lin, Y.-K. Ko, L. Sui, J.C. Raymond, G.A. Stenborg, Y. Jiang, S. Zhao, S. Mancuso, Direct Observations of the Magnetic Reconnection Site of an Eruption on 2003 November 18. Astrophys. J. 622, 1251-1264 (2005). doi:10.1086/428110

M.L. Lister, M.H. Cohen, D.C. Homan, M. Kadler, K.I. Kellermann, Y.Y. Kovalev, E. Ros, T. Savolainen, J.A. Zensus, MOJAVE: Monitoring of Jets in Active Galactic Nuclei with VLBA Experiments. VI. Kinematics Analysis of a Complete Sample of Blazar Jets. Astron. J. 138, 1874-1892 (2009). doi:10.1088/0004-6256/138/6/1874

W. Liu, H. Li, L. Yin, B.J. Albright, K.J. Bowers, E.P. Liang, Particle energization in 3D magnetic reconnection of relativistic pair plasmas. Physics of Plasmas 18(5), 052105 (2011). doi:10.1063/1.3589304

Y.-H. Liu, J.F. Drake, M. Swisdak, The structure of the magnetic reconnection exhaust boundary. Physics of Plasmas 19(2), 022110 (2012). doi:10.1063/1.3685755

N.F. Loureiro, A.A. Schekochihin, S.C. Cowley, Instability of current sheets and formation of plasmoid chains. Physics of Plasmas 14(10), 100703 (2007). doi:10.1063/1.2783986

N.F. Loureiro, R. Samtaney, A.A. Schekochihin, D.A. Uzdensky, Magnetic reconnection and stochastic plasmoid chains in high-Lundquist-number plasmas. Physics of Plasmas 19(4), 042303 (2012). doi:10.1063/1.3703318

Y.E. Lyubarskii, A model for the energetic emission from pulsars. Astron. Astrophys. 311, 172-178 (1996)

Y.E. Lyubarskij, Energy release in strongly magnetized relativistic winds. Soviet Astronomy Letters 18, $356(1992)$

Y. Lyubarsky, J.G. Kirk, Reconnection in a Striped Pulsar Wind. Astrophys. J. 547, 437448 (2001). doi:10.1086/318354

Y. Lyubarsky, M. Liverts, Particle Acceleration in the Driven Relativistic Reconnection. Astrophys. J. 682, 1436-1442 (2008). doi:10.1086/589640

Y.E. Lyubarsky, The termination shock in a striped pulsar wind. Mon. Not. R. Astron. Soc. 345, 153-160 (2003). doi:10.1046/j.1365-8711.2003.06927.x

Y.E. Lyubarsky, On the relativistic magnetic reconnection. Mon. Not. R. Astron. Soc. 358, 113-119 (2005). doi:10.1111/j.1365-2966.2005.08767.x

Y.E. Lyubarsky, Transformation of the Poynting flux into kinetic energy in relativistic jets. Mon. Not. R. Astron. Soc. 402, 353-361 (2010). doi:10.1111/j.1365-2966.2009.15877.x

Y.E. Lyubarsky, Highly magnetized region in pulsar wind nebulae and origin of the Crab gamma-ray flares. Mon. Not. R. Astron. Soc. 427, 1497-1502 (2012). doi:10.1111/j.13652966.2012.22097.x

M. Lyutikov, The electromagnetic model of gamma-ray bursts. New Journal of Physics 8 , 119 (2006). doi:10.1088/1367-2630/8/7/119

J.C. McKinney, D.A. Uzdensky, A reconnection switch to trigger gamma-ray burst jet dissipation. Mon. Not. R. Astron. Soc. 419, 573-607 (2012). doi:10.1111/j.13652966.2011.19721.x

M. Melzani, C. Winisdoerffer, R. Walder, D. Folini, J.M. Favre, S. Krastanov, P. Messmer, Apar-T: code, validation, and physical interpretation of particle-in-cell results. Astron. Astrophys. 558, 133 (2013). doi:10.1051/0004-6361/201321557

M. Melzani, R. Walder, D. Folini, C. Winisdoerffer, J.M. Favre, The energetics of relativistic magnetic reconnection: ion-electron repartition and particle distribution hardness. ArXiv e-prints (2014)

F.C. Michel, Magnetic structure of pulsar winds. Astrophys. J. 431, 397-401 (1994). doi:10.1086/174493

A. Mignone, E. Striani, M. Tavani, A. Ferrari, Modelling the kinked jet of the Crab nebula. Mon. Not. R. Astron. Soc. 436, 1102-1115 (2013). doi:10.1093/mnras/stt1632

Y. Mizuno, Y. Lyubarsky, K.-I. Nishikawa, P.E. Hardee, Three-dimensional Relativistic 
Magnetohydrodynamic Simulations of Current-driven Instability. II. Relaxation of Pulsar Wind Nebula. Astrophys. J. 728, 90 (2011). doi:10.1088/0004-637X/728/2/90

K. Nalewajko, D. Giannios, M.C. Begelman, D.A. Uzdensky, M. Sikora, Radiative properties of reconnection-powered minijets in blazars. Mon. Not. R. Astron. Soc. 413, 333-346 (2011). doi:10.1111/j.1365-2966.2010.18140.x

R. Narayan, T. Piran, Variability in blazars: clues from PKS 2155-304. Mon. Not. R. Astron. Soc. 420, 604-612 (2012). doi:10.1111/j.1365-2966.2011.20069.x

C. Nodes, G.T. Birk, H. Lesch, R. Schopper, Particle acceleration in three-dimensional tearing configurations. Physics of Plasmas 10, 835-844 (2003). doi:10.1063/1.1542612

M. Øieroset, T.D. Phan, J.P. Eastwood, M. Fujimoto, W. Daughton, M.A. Shay, V. Angelopoulos, F.S. Mozer, J.P. McFadden, D.E. Larson, K.-H. Glassmeier, Direct Evidence for a Three-Dimensional Magnetic Flux Rope Flanked by Two Active Magnetic Reconnection X Lines at Earth's Magnetopause. Physical Review Letters 107(16), 165007 (2011). doi:10.1103/PhysRevLett.107.165007

M. Oka, T.-D. Phan, S. Krucker, M. Fujimoto, I. Shinohara, Electron Acceleration by Multi-Island Coalescence. Astrophys. J. 714, 915-926 (2010). doi:10.1088/0004$637 \mathrm{X} / 714 / 1 / 915$

V. Olshevsky, G. Lapenta, S. Markidis, Energetics of kinetic reconnection in a three-dimensional null-point cluster. Phys. Rev. Lett. 111, $045002 \quad$ (2013). doi:10.1103/PhysRevLett.111.045002. http://link.aps.org/doi/10.1103/PhysRevLett.111.045002

H.K. Park, N.C. Luhmann Jr., A.J.H. Donné, I.G.J. Classen, C.W. Domier, E. Mazzucato, T. Munsat, M.J. van de Pol, Z. Xia, Observation of High-Field-Side Crash and Heat Transfer during Sawtooth Oscillation in Magnetically Confined Plasmas. Physical Review Letters 96(19), 195003 (2006). doi:10.1103/PhysRevLett.96.195003

J. Pétri, High-energy emission from the pulsar striped wind: a synchrotron model for gammaray pulsars. Mon. Not. R. Astron. Soc. 424, 2023-2027 (2012). doi:10.1111/j.13652966.2012.21350.x

J. Pétri, J.G. Kirk, Growth rates of the Weibel and tearing mode instabilities in a relativistic pair plasma. Plasma Physics and Controlled Fusion 49, 1885-1896 (2007). doi:10.1088/0741-3335/49/11/009

J. Pétri, Y. Lyubarsky, Magnetic reconnection at the termination shock in a striped pulsar wind. Astron. Astrophys. 473, 683-700 (2007). doi:10.1051/0004-6361:20066981

H.E. Petschek, Magnetic Field Annihilation. NASA Special Publication 50, 425 (1964)

B.G. Piner, A.B. Pushkarev, Y.Y. Kovalev, C.J. Marvin, J.G. Arenson, P. Charlot, A.L. Fey, A. Collioud, P.A. Voitsik, Relativistic Jets in the Radio Reference Frame Image Database. II. Blazar Jet Accelerations from the First 10 Years of Data (1994-2003). Astrophys. J. 758, 84 (2012). doi:10.1088/0004-637X/758/2/84

D.I. Pontin, Three-dimensional magnetic reconnection regimes: A review. Advances in Space Research 47, 1508-1522 (2011). doi:10.1016/j.asr.2010.12.022

O. Porth, S.S. Komissarov, R. Keppens, Solution to the sigma problem of pulsar wind nebulae. Mon. Not. R. Astron. Soc. 431, 48-52 (2013). doi:10.1093/mnrasl/slt006

O. Porth, S.S. Komissarov, R. Keppens, Three-dimensional magnetohydrodynamic simulations of the Crab nebula. Mon. Not. R. Astron. Soc. 438, 278-306 (2014). doi:10.1093/mnras/stt2176

A.B. Pushkarev, Y.Y. Kovalev, M.L. Lister, T. Savolainen, Jet opening angles and gammaray brightness of AGN. Astron. Astrophys. 507, 33-36 (2009). doi:10.1051/0004$6361 / 200913422$

R. Samtaney, N.F. Loureiro, D.A. Uzdensky, A.A. Schekochihin, S.C. Cowley, Formation of Plasmoid Chains in Magnetic Reconnection. Physical Review Letters 103(10), 105004 (2009). doi:10.1103/PhysRevLett.103.105004

T. Savolainen, D.C. Homan, T. Hovatta, M. Kadler, Y.Y. Kovalev, M.L. Lister, E. Ros, J.A. Zensus, Relativistic beaming and gamma-ray brightness of blazars. Astron. Astrophys. 512, 24 (2010). doi:10.1051/0004-6361/200913740

L. Sironi, A. Spitkovsky, Acceleration of Particles at the Termination Shock of a Relativistic Striped Wind. Astrophys. J. 741, 39 (2011a). doi:10.1088/0004-637X/741/1/39

L. Sironi, A. Spitkovsky, Particle Acceleration in Relativistic Magnetized Collisionless Electron-Ion Shocks. Astrophys. J. 726, 75 (2011b). doi:10.1088/0004-637X/726/2/75 
L. Sironi, A. Spitkovsky, Particle-in-cell simulations of shock-driven reconnection in relativistic striped winds. Computational Science and Discovery 5(1), 014014 (2012). doi:10.1088/1749-4699/5/1/014014

L. Sironi, A. Spitkovsky, Relativistic Reconnection: An Efficient Source of Non-thermal Particles. Astrophys. J. Lett. 783, 21 (2014). doi:10.1088/2041-8205/783/1/L21

L. Sironi, A. Spitkovsky, J. Arons, The Maximum Energy of Accelerated Particles in Relativistic Collisionless Shocks. Astrophys. J. 771, 54 (2013). doi:10.1088/0004$637 \mathrm{X} / 771 / 1 / 54$

T.W. Speiser, Particle Trajectories in Model Current Sheets, 1, Analytical Solutions. J. Geophys. Res. 70, 4219-4226 (1965). doi:10.1029/JZ070i017p04219

Y.T. Tanaka, Ł. Stawarz, D.J. Thompson, F. D'Ammando, S.J. Fegan, B. Lott, D.L. Wood, C.C. Cheung, J. Finke, S. Buson, L. Escande, S. Saito, M. Ohno, T. Takahashi, D. Donato, J. Chiang, M. Giroletti, F.K. Schinzel, G. Iafrate, F. Longo, S. Ciprini, Fermi Large Area Telescope Detection of Bright $\gamma$-Ray Outbursts from the Peculiar Quasar 4C +21.35. Astrophys. J. 733, 19 (2011). doi:10.1088/0004-637X/733/1/19

M. Tavani, A. Bulgarelli, V. Vittorini, A. Pellizzoni, E. Striani, P. Caraveo, M.C. Weisskopf, A. Tennant, G. Pucella, A. Trois, E. Costa, Y. Evangelista, C. Pittori, F. Verrecchia, E. Del Monte, R. Campana, M. Pilia, A. De Luca, I. Donnarumma, D. Horns, C. Ferrigno, C.O. Heinke, M. Trifoglio, F. Gianotti, S. Vercellone, A. Argan, G. Barbiellini, P.W. Cattaneo, A.W. Chen, T. Contessi, F. D'Ammando, G. DeParis, G. Di Cocco, G. Di Persio, M. Feroci, A. Ferrari, M. Galli, A. Giuliani, M. Giusti, C. Labanti, I. Lapshov, F. Lazzarotto, P. Lipari, F. Longo, F. Fuschino, M. Marisaldi, S. Mereghetti, E. Morelli, E. Moretti, A. Morselli, L. Pacciani, F. Perotti, G. Piano, P. Picozza, M. Prest, M. Rapisarda, A. Rappoldi, A. Rubini, S. Sabatini, P. Soffitta, E. Vallazza, A. Zambra, D. Zanello, F. Lucarelli, P. Santolamazza, P. Giommi, L. Salotti, G.F. Bignami, Discovery of Powerful Gamma-Ray Flares from the Crab Nebula. Science 331, 736 (2011). doi:10.1126/science. 1200083

F. Tavecchio, J. Becerra-Gonzalez, G. Ghisellini, A. Stamerra, G. Bonnoli, L. Foschini, L. Maraschi, On the origin of the $\gamma$-ray emission from the flaring blazar PKS $1222+216$. Astron. Astrophys. 534, 86 (2011). doi:10.1051/0004-6361/201117204

C.M. Urry, P. Padovani, Unified Schemes for Radio-Loud Active Galactic Nuclei. Publ. Astron. Soc. Pac. 107, 803 (1995). doi:10.1086/133630

D.A. Uzdensky, R.M. Kulsrud, Two-dimensional numerical simulation of the resistive reconnection layer. Physics of Plasmas 7, 4018-4030 (2000). doi:10.1063/1.1308081

D.A. Uzdensky, A. Spitkovsky, Physical Conditions in the Reconnection Layer in Pulsar Magnetospheres. Astrophys. J. 780, 3 (2014). doi:10.1088/0004-637X/780/1/3

D.A. Uzdensky, B. Cerutti, M.C. Begelman, Reconnection-powered Linear Accelerator and Gamma-Ray Flares in the Crab Nebula. Astrophys. J. Lett. 737, 40 (2011). doi:10.1088/2041-8205/737/2/L40

D.A. Uzdensky, N.F. Loureiro, A.A. Schekochihin, Fast Magnetic Reconnection in the Plasmoid-Dominated Regime. Physical Review Letters 105(23), 235002 (2010). doi:10.1103/PhysRevLett.105.235002

G.R. Werner, D.A. Uzdensky, B. Cerutti, K. Nalewajko, M.C. Begelman, The extent of power-law energy spectra in collisionless relativistic magnetic reconnection in pair plasmas. ArXiv e-prints (2014)

L. Yin, W. Daughton, H. Karimabadi, B.J. Albright, K.J. Bowers, J. Margulies, Three-Dimensional Dynamics of Collisionless Magnetic Reconnection in Large-Scale Pair Plasmas. Physical Review Letters 101(12), 125001 (2008). doi:10.1103/PhysRevLett.101.125001

O. Zanotti, M. Dumbser, Numerical simulations of high Lundquist number relativistic magnetic reconnection. Mon. Not. R. Astron. Soc. 418, 1004-1011 (2011). doi:10.1111/j.1365-2966.2011.19551.x

S. Zenitani, M. Hesse, Self-Regulation of the Reconnecting Current Layer in Relativistic Pair Plasma Reconnection. Astrophys. J. 684, 1477-1485 (2008). doi:10.1086/590425

S. Zenitani, M. Hoshino, The Generation of Nonthermal Particles in the Relativistic Magnetic Reconnection of Pair Plasmas. Astrophys. J. Lett. 562, 63-66 (2001). doi:10.1086/337972

S. Zenitani, M. Hoshino, Relativistic Particle Acceleration in a Folded Current Sheet. As- 
trophys. J. Lett. 618, 111-114 (2005). doi:10.1086/427873

S. Zenitani, M. Hoshino, Particle Acceleration and Magnetic Dissipation in Relativistic Current Sheet of Pair Plasmas. Astrophys. J. 670, 702-726 (2007). doi:10.1086/522226

S. Zenitani, M. Hoshino, The Role of the Guide Field in Relativistic Pair Plasma Reconnection. Astrophys. J. 677, 530-544 (2008). doi:10.1086/528708

B. Zhang, H. Yan, The Internal-collision-induced Magnetic Reconnection and Turbulence (ICMART) Model of Gamma-ray Bursts. Astrophys. J. 726, 90 (2011). doi:10.1088/0004-637X/726/2/90 